\title{
Banach-Saks properties in symmetric spaces of measurable operators
}

\author{
by \\ P. G. Dodds, T. K. Dodds and F. A. Sukochev (Bedford Park)
}

\begin{abstract}
We study Banach-Saks properties in symmetric spaces of measurable operators. A principal result shows that if the symmetric Banach function space $E$ on the positive semiaxis with the Fatou property has the Banach-Saks property then so also does the non-commutative space $E(\mathcal{M}, \tau)$ of $\tau$-measurable operators affiliated with a given semifinite von Neumann algebra $(\mathcal{M}, \tau)$.
\end{abstract}

0. Introduction. A Banach space is said to have the Banach-Saks property if each weakly null sequence contains a subsequence whose arithmetic means converge strongly to zero. It is a classical result of Banach and Saks [BS], and Banach and Mazur [Ba], that the spaces $L_{p}[0,1), 1<p<\infty$, have this property. In the case that $p>2$, this result also follows directly from the work of Kadec and Pełczyński [KP]. While it is a well known result of Kakutani (see [Di]) that any uniformly convex Banach space has the Banach-Saks property, it was shown by Szlenk [Sz] that the non-uniformly convex Banach space $L_{1}[0,1)$ also has the Banach-Saks property. More recently, the Banach-Saks property has been studied in the framework of rearrangement invariant spaces [DSS], where it has been shown, in particular, that any separable Orlicz or Lorentz space on the unit interval has the Banach-Saks property. While any Banach space with the Banach-Saks property is necessarily separable, separability on its own is not a sufficient condition for the validity of the Banach-Saks property. Indeed, it is shown in [DSS] that the separable part of each non-separable Orlicz or Lorentz space fails to have the Banach-Saks property, as do certain separable Marcinkiewicz spaces.

From a somewhat different viewpoint, the Banach-Saks property has been studied in the setting of unitary matrix ideals (Schatten ideals) by

2000 Mathematics Subject Classification: Primary 46E30; Secondary 46L51, 46L52.

Key words and phrases: Banach-Saks property, symmetric Banach function space, measurable operator.

Research of P. G. Dodds partially supported by the Australian Research Council. 
Arazy [Ar]. If $E$ is a separable symmetric sequence space, then it was shown in $[\mathrm{Ar}]$ that $E$ has the Banach-Saks property if and only if the associated unitary matrix space $\mathcal{C}_{E}$ has the Banach-Saks property. Here $\mathcal{C}_{E}$ is the space of all compact operators $x$ on some separable Hilbert space for which $s(x) \in E$ with norm given by $\|x\|_{C_{E}}=\|s(x)\|_{E}$, where $s(x)=\left\{s_{n}(x)\right\}_{n=1}^{\infty}$ is the sequence of $s$-numbers of $x$. The approach of [Ar] is based on the study of shell block basic sequences in unitary matrix spaces. The purpose of this paper is to show that this result of Arazy continues to hold in the more general setting of symmetric spaces $E(\mathcal{M}, \tau)$ of measurable operators associated with a symmetric Banach function space $E$ on the positive semiaxis.

A principal result of the paper (Theorem 2.13) shows that if the von Neumann algebra $(\mathcal{M}, \tau)$ is non-atomic, and if $E$ has the Fatou property, then $E(\mathcal{M}, \tau)$ has the Banach-Saks property if and only if $E(\mathcal{M}, \tau)$ has the Banach-Saks property for disjointly supported sequences. This extends a similar result in the commutative setting given in [DSS], where it is shown that the assumption that $E$ has the Fatou property cannot be omitted, and this is in strong contrast to the situation in separable symmetric sequence spaces, in which the Banach-Saks property and that for disjointly supported sequences are always equivalent. It follows readily from Theorem 2.13 that if the separable symmetric Banach function space $E$ on the positive semiaxis with the Fatou property has the Banach-Saks property then so also does the non-commutative space $E(\mathcal{M}, \tau)$ for all semifinite $(\mathcal{M}, \tau)$ (Theorem 2.14). Specialisation to the von Neumann algebra of all bounded linear operators on a separable Hilbert space recovers the results of Arazy for unitary matrix spaces associated with separable symmetric sequence spaces with the Fatou property. However, our methods are completely different from those of $[\mathrm{Ar}]$, and our approach is based on the systematic study of Banach-Saks type properties in (commutative) rearrangement-invariant Banach function spaces given in [DSS], combined with the study of (non-commutative) symmetric spaces of measurable operators using methods of real analysis [FK], [CS], [CKS], [CSS], [DDP1-3]. A principal ingredient is a decomposition theorem (Proposition 2.7): if the von Neumann algebra $(\mathcal{M}, \tau)$ is non-atomic and if $E$ is a separable space with the Fatou property, then each bounded sequence in $E(\mathcal{M}, \tau)$ contains a subsequence which is a norm perturbation of the sum of an equimeasurable sequence and a sequence which is two-sided disjointly supported and which converges to zero for the measure topology. The ideas here go back to the paper $[\mathrm{KP}]$ in classical (commutative) $L_{p}$-spaces.

As a by-product of our approach, we show (Theorem 2.8) that if $E$ is separable and has the Fatou property, then each bounded sequence in $E(\mathcal{M}, \tau)$ contains a subsequence with the property that the Cesàro means of each further subsequence are convergent in the measure topology. This 
is a non-commutative analogue of a well known theorem of Komlós [Ko]. Finally, the difference between the purely atomic type I setting studied by Arazy and the more general setting including non-atomic algebras studied in the present paper yields the interesting consequence that if $\Phi$ is an Orlicz function for which the corresponding Orlicz space $E_{\Phi}$ is non-separable, if $(\mathcal{M}, \tau)$ is non-atomic and if $(\mathcal{N}, \sigma)$ is the von Neumann algebra of all bounded linear operators on a separable Hilbert space equipped with the canonical trace $\sigma$, then $E_{\Phi}(\mathcal{M}, \tau)$ does not embed isomorphically in the unitary matrix space $E_{\Phi}(\mathcal{N}, \sigma)=\mathcal{C}_{E_{\Phi}}$.

In the third section of the paper, we consider the (so-called) $p$-BanachSaks and strong $p$-Banach-Saks properties studied in [HRS], [RX] in the setting of non-commutative $L_{p^{-}}$-spaces. A principal result (Theorem 3.9) is that if the commutative space $E$ is $p$-convex and $q$-concave for some $1<p<2 \leq q<\infty$ and if $(\mathcal{M}, \tau)$ is non-atomic, then each weakly null, $E$-equiintegrable sequence in $E(\mathcal{M}, \tau)$ contains a strong $p$-Banach-Saks subsequence. We show further (Theorem 3.14) that if the Lorentz function $\psi$ is regular, then a closed subspace $X$ of the non-commutative Lorentz space $\Lambda_{\psi, p}(\mathcal{M}, \tau), 1 \leq p<\infty$, has the strong $p$-Banach-Saks property if and only if $X$ contains no isomorph of $l_{p}$. These results are new, even in the commutative setting, and extend similar results in [HRS], [RX] for the special case of non-commutative $L_{p}$-spaces.

1. Preliminaries. In this section, we collect some of the basic facts and notation that will be used in this paper. We denote by $\mathcal{M}$ a semifinite von Neumann algebra on the Hilbert space $\mathcal{H}$, with a fixed faithful and normal semifinite trace $\tau$. The identity in $\mathcal{M}$ is denoted by 1 and we denote by $\mathcal{P}$ the complete lattice of all (self-adjoint) projections in $\mathcal{M}$. A linear operator $x: \operatorname{dom}(x) \rightarrow \mathcal{H}$, with domain $\operatorname{dom}(x) \subseteq \mathcal{H}$, is said to be affiliated with $\mathcal{M}$ if $u x=x u$ for all unitary $u$ in the commutant $\mathcal{M}^{\prime}$ of $\mathcal{M}$. The closed and densely defined operator $x$ affiliated with $\mathcal{M}$ is called $\tau$-measurable if for every $\varepsilon>0$ there exists an orthogonal projection $p \in \mathcal{M}$ such the $p(\mathcal{H}) \subseteq \operatorname{dom}(x)$ and $\tau(\mathbf{1}-p)<\varepsilon$. The collection of all $\tau$-measurable operators is denoted by $\widetilde{\mathcal{M}}$. With the sum and product defined as the respective closures of the algebraic sum and product, $\widetilde{\mathcal{M}}$ is a $*$-algebra. For $\varepsilon, \delta>0$ we denote by $N(\varepsilon, \delta)$ the set of all $x \in \widetilde{\mathcal{M}}$ for which there exists an orthogonal projection $p \in \mathcal{M}$ such that $p(\mathcal{H}) \subseteq \operatorname{dom}(x),\|x p\|_{\infty} \leq \varepsilon$ and $\tau(\mathbf{1}-p) \leq \delta$, where $\|\cdot\|_{\infty}$ denotes the usual operator norm. The sets $\{N(\varepsilon, \delta): \varepsilon, \delta>0\}$ form a base at 0 for a metrisable Hausdorff topology in $\widetilde{\mathcal{M}}$, which is called the measure topology. Equipped with the measure topology, $\widetilde{\mathcal{M}}$ is a complete topological $*$-algebra. These facts and their proofs can be found in the papers $[\mathrm{Ne}]$ and $[\mathrm{Te}]$. For standard facts concerning von Neumann algebras, we refer to $[\mathrm{SZ}],[\mathrm{Ta}]$. 
We recall the notion of generalised singular value function [FK]. Given a self-adjoint operator $x$ in $\mathcal{H}$ we denote by $e^{x}(\cdot)$ the spectral measure of $x$. Now assume that $x \in \widetilde{\mathcal{M}}$. Then $e^{|x|}(B) \in \mathcal{M}$ for all Borel sets $B \subseteq \mathbb{R}$, and there exists $s>0$ such that $\tau\left(e^{|x|}(s, \infty)\right)<\infty$. For $x \in \widetilde{\mathcal{M}}$ and $t \geq 0$ we define

$$
\mu_{t}(x)=\inf \left\{s \geq 0: \tau\left(e^{|x|}(s, \infty)\right) \leq t\right\} .
$$

The function $\mu(x):[0, \infty) \rightarrow[0, \infty]$ is called the generalised singular value function (or decreasing rearrangement) of $x$; note that $\mu_{t}(x)<\infty$ for all $t>0$. For the basic properties of this singular value function we refer the reader to $[\mathrm{FK}]$; some additional properties can be found in [DDP1,2]. We note that a sequence $\left\{x_{n}\right\} \subseteq \widetilde{\mathcal{M}}$ converges to 0 for the measure topology if and only if $\mu_{t}\left(x_{n}\right) \rightarrow 0$ for all $t>0$.

If we consider $\mathcal{M}=L_{\infty}\left(\mathbb{R}^{+}, m\right)$, where $m$ denotes Lebesgue measure on the positive half-line $\mathbb{R}^{+}$, as an Abelian von Neumann algebra acting via multiplication on the Hilbert space $\mathcal{H}=L_{2}\left(\mathbb{R}^{+}, m\right)$, with the trace given by integration with respect to $m$, then it is easy to see that $\widetilde{\mathcal{M}}$ consists of all measurable functions on $\mathbb{R}^{+}$which are bounded except on a set of finite measure, and that for $f \in \widetilde{\mathcal{M}}$, the generalised singular value function $\mu(f)$ is precisely the decreasing rearrangement of the function $|f|$ (and in this setting, $\mu(f)$ is frequently denoted by $f^{*}$ ). If $\mathcal{M}=\mathcal{L}(\mathcal{H})$ and $\tau$ is the standard trace, then it is not difficult to see that $\widetilde{\mathcal{M}}=\mathcal{M}$ and that the measure topology coincides with the operator norm topology. In this case, $x \in \mathcal{M}$ is compact if and only if $\lim _{t \rightarrow \infty} \mu_{t}(x)=0$; moreover,

$$
\mu_{n}(x)=\mu_{t}(x), \quad t \in[n, n+1), n=0,1,2, \ldots,
$$

and the sequence $\left\{\mu_{n}(x)\right\}_{n=0}^{\infty}$ is just the sequence of eigenvalues of $|x|$ in non-increasing order and counted according to multiplicity.

By $L^{0}\left(\mathbb{R}^{+}, m\right)$ we denote the space of all $\mathbb{C}$-valued Lebesgue measurable functions on $\mathbb{R}^{+}$(with identification $m$-a.e.). A Banach space $\left(E,\|\cdot\|_{E}\right)$, where $E \subseteq L^{0}\left(\mathbb{R}^{+}, m\right)$ is called a rearrangement-invariant Banach function space if it follows from $f \in E, g \in L^{0}\left(\mathbb{R}^{+}, m\right)$ and $\mu(g) \leq \mu(f)$ that $g \in E$ and $\|g\|_{E} \leq\|f\|_{E}$. Furthermore, $\left(E,\|\cdot\|_{E}\right)$ is called a symmetric Banach function space if it has the additional property that $f, g \in E$ and $g \nVdash f$ imply that $\|g\|_{E} \leq\|f\|_{E}$. The symmetric Banach function space $\left(E,\|\cdot\|_{E}\right)$ is called fully symmetric if $f \in E, g \in L^{0}\left(\mathbb{R}^{+}, m\right)$ and $g \preccurlyeq f$ imply $g \in E$ and $\|g\|_{E} \leq\|f\|_{E}$. It is shown in [DDP2,3] that $E$ is fully symmetric if and only if $E(\mathcal{M}, \tau)$ is an exact interpolation space for the couple $\left(L^{1}(\mathcal{M}, \tau), \mathcal{M}\right)$. Here $g \preccurlyeq f$ denotes submajorisation in the sense of Hardy-Littlewood-Pólya:

$$
\int_{0}^{t} \mu_{s}(g) d s \leq \int_{0}^{t} \mu_{s}(f) d s \quad \text { for all } t>0 .
$$

For the general theory of rearrangement-invariant Banach function spaces, 
we refer the reader to [KPS], [BeS], [LT], although in the latter two references the class of function spaces considered is more restrictive.

Given a semifinite von Neumann algebra $(\mathcal{M}, \tau)$ and a symmetric Banach function space $\left(E,\|\cdot\|_{E}\right)$ on $\left(\mathbb{R}^{+}, m\right)$, we define the corresponding noncommutative space $E(\mathcal{M}, \tau)$ by setting

$$
E(\mathcal{M}, \tau)=\{x \in \widetilde{\mathcal{M}}: \mu(x) \in E\} .
$$

Equipped with the norm $\|x\|_{E(\mathcal{M}, \tau)}:=\|\mu(x)\|_{E}$, the space $(E(\mathcal{M}, \tau)$, $\left.\|\cdot\|_{E(\mathcal{M}, \tau)}\right)$ is a Banach space and is called the (non-commutative) symmetric space associated with $(\mathcal{M}, \tau)$ corresponding to $\left(E,\|\cdot\|_{E}\right)$. An extensive discussion of the various properties of such spaces can be found in [DDP1-3]. The Köthe dual $E(\mathcal{M}, \tau)^{\times}$is defined to be the set of all $x \in \widetilde{\mathcal{M}}$ such that $x y \in L_{1}(\mathcal{M}, \tau)$ for all $y \in E(\mathcal{M}, \tau)$. With the norm defined by setting

$$
\begin{array}{r}
\|x\|_{E(\mathcal{M}, \tau)^{\times}}:=\sup \left\{\tau(|x y|): y \in E(\mathcal{M}, \tau),\|y\|_{E(\mathcal{M}, \tau)} \leq 1\right\}, \\
x \in E(\mathcal{M}, \tau)^{\times},
\end{array}
$$

the Köthe dual $E(\mathcal{M}, \tau)^{\times}$is a Banach space. Basic properties of Köthe duality, in the commutative setting, may be found in [KPS], [BeS] (where the Köthe dual is called the associate space). In the non-commutative setting, the reader is referred to [DDP3], where it is shown in particular that if $E$ is symmetric, then the space $\left(E(\mathcal{M}, \tau)^{\times},\|\cdot\|_{E(\mathcal{M}, \tau)^{\times}}\right)$may be identified with the space $\left(E^{\times}(\mathcal{M}, \tau),\|\cdot\|_{E^{\times}(\mathcal{M}, \tau)}\right)$.

If $E$ is a symmetric Banach function space on $\mathbb{R}^{+}$, then $E$ is separable if and only if the norm on $E$ is order continuous in the sense that $0 \leq f_{\sigma} \downarrow_{\sigma} 0$ in $E$ implies that $\left\|f_{\tau}\right\|_{E} \downarrow_{\sigma} 0$. In this case, the norm is order continuous (in the obvious sense) on the non-commutative space $E(\mathcal{M}, \tau)$. The symmetric space $E$ is separable if and only if the Banach dual $E^{*}$ coincides with the Köthe dual $E^{\times}$of $E$. In this case, the dual space $E^{*}$ is a symmetric Banach function space on $\mathbb{R}^{+}$and it follows from [DDP3, Theorems 5.6, $5.11]$ that the Banach dual of the space $\left(E(\mathcal{M}, \tau),\|\cdot\|_{E(\mathcal{M}, \tau)}\right)$ is the space $\left(E^{*}(\mathcal{M}, \tau),\|\cdot\|_{E^{*}(\mathcal{M}, \tau)}\right)$. If $E$ is separable, then $E$ is fully symmetric in the sense that $f \in E, g \in L^{0}\left(\mathbb{R}^{+}, m\right)$ and $g \ll f$ imply that $g \in E$ and $\|g\|_{E} \leq\|f\|_{E}$.

We set

$$
\begin{gathered}
\widetilde{\mathcal{M}}_{0}:=\left\{x \in \widetilde{\mathcal{M}}: \mu_{t}(x) \rightarrow 0 \text { as } t \rightarrow \infty\right\}, \\
L_{0}(\mathcal{M}, \tau):=\widetilde{\mathcal{M}}_{0} \cap\left(L_{1}(\mathcal{M}, \tau)+\mathcal{M}\right),
\end{gathered}
$$

and make the obvious remark that $\widetilde{\mathcal{M}}_{0}$ coincides with $\widetilde{\mathcal{M}}$ if $\tau(\mathbf{1})<\infty$. For brevity, we denote the space $L_{0}\left(L_{\infty}\left(\mathbb{R}^{+}, m\right)\right)$ by $L_{0}[0, \infty)$. By [DDP3, Proposition 2.7], the closure of the space $L_{1}(\mathcal{M}, \tau) \cap \mathcal{M}$ in the space $L_{1}(\mathcal{M}, \tau)+\mathcal{M}$ coincides with $L_{0}(\mathcal{M}, \tau)$. Moreover, for any symmetric Banach function 
space $E$ on $\mathbb{R}^{+}$, the inclusions

$$
L_{1}(\mathcal{M}, \tau) \cap \mathcal{M} \subseteq E(\mathcal{M}, \tau), \quad E^{\times}(\mathcal{M}, \tau) \subseteq L_{1}(\mathcal{M}, \tau)+\mathcal{M}
$$

hold with continuous embeddings. It is clear that the embedding $E \subseteq$ $L_{0}[0, \infty)$ implies the embedding $E(\mathcal{M}, \tau) \subseteq \widetilde{\mathcal{M}}_{0}$. We remark that if $E$ is separable then $E(\mathcal{M}, \tau) \subseteq L_{0}(\mathcal{M}, \tau)$.

If $\mathcal{N}$ is a von Neumann subalgebra of $\mathcal{M}$, then $\mathcal{N}$ will be called proper if the restriction $\tau_{\mathcal{N}}$ of $\tau$ to $\mathcal{N}$ is again semifinite. The first part of the result below may be found in [DDP2, Theorem 3.5]. The second assertion follows by a straightforward modification of the arguments of [CS, Lemma 4.1] and [CKS, Lemma 1.3].

Proposition 1.1. If $x \in \widetilde{\mathcal{M}}_{0}$, then there exists a proper von Neumann subalgebra $M_{x} \subseteq L_{\infty}\left(\mathbb{R}^{+}, m\right)$ with $\mu(x) \in \widetilde{M}_{x}$, a proper commutative subalgebra $\mathcal{M}_{x} \subseteq \mathcal{M}$ and a positive rearrangement-preserving algebra *-isomorphism $J_{x}$ of $\widetilde{M}_{x}$ onto $\widetilde{\mathcal{M}}_{x}$ whose restriction to the lattice of projections of $M_{x}$ is a Boolean algebra isomorphism onto the lattice of projections of $\mathcal{M}_{x}$ and for which

$$
J_{x}(\mu(x))=|x| .
$$

If , in addition, $x$ is self-adjoint, and if $(\mathcal{M}, \tau)$ is non-atomic, then $\mathcal{M}_{x}$ may be taken to be a non-atomic maximal Abelian von Neumann algebra containing the spectral resolution of $X$, and the commutative von Neumann algebra $M_{x}$ may be taken to be the commutative von Neumann algebra $L_{\infty}\left(\mathbb{R}^{+}, m\right)$.

If $\mathcal{N} \subseteq \mathcal{M}$ is a proper von Neumann subalgebra, then the conditional expectation

$$
\mathcal{E}_{\mathcal{N}}: L_{1}(\mathcal{M}, \tau)+\mathcal{M} \rightarrow L_{1}\left(\mathcal{N}, \tau_{\mathcal{N}}\right)+\mathcal{N}
$$

is defined as in the commutative setting via the equality

$$
\tau_{\mathcal{N}}\left(\mathcal{E}_{\mathcal{N}}(x) y\right)=\tau(x y), \quad x \in L_{1}(\mathcal{M}, \tau)+\mathcal{M}, y \in L_{1}\left(\mathcal{N}, \tau_{\mathcal{N}}\right) \cap \mathcal{N},
$$

and an appeal to the fact that the spaces $L_{1}\left(\mathcal{N}, \tau_{\mathcal{N}}\right)+\mathcal{N}, L_{1}\left(\mathcal{N}, \tau_{\mathcal{N}}\right) \cap \mathcal{N}$ are dual in the sense of Köthe. See, for example, [DDP3, Theorem 5.6].

The following is proved in [DDS, Lemma 5.1].

Lemma 1.2. Let $\mathcal{N} \subseteq \mathcal{M}$ be a proper von Neumann subalgebra. If $E$ is a fully symmetric Banach function space on $\mathbb{R}^{+}$, then $\mathcal{E}_{\mathcal{N}}(x) \in E\left(\mathcal{N}, \tau_{\mathcal{N}}\right)$ for all $x \in E(\mathcal{M}, \tau)$ and

$$
\tau_{\mathcal{N}}\left(\mathcal{E}_{\mathcal{N}}(x) y\right)=\tau(x y), \quad x \in E(\mathcal{M}, \tau), y \in E\left(\mathcal{N}, \tau_{\mathcal{N}}\right)^{*}=E^{*}\left(\mathcal{N}, \tau_{\mathcal{N}}\right) .
$$

2. The Banach-Saks property. It will be convenient to adopt the following terminology. 
Definition 2.1. Let $X$ be a Banach space.

(a) A weakly null sequence $\left\{x_{n}\right\}_{n=1}^{\infty} \subseteq X$ is said to be a Banach-Saks sequence if

$$
\lim _{n \rightarrow \infty} n^{-1}\left\|\sum_{j=1}^{n} y_{j}\right\|=0
$$

for all subsequences $\left\{y_{j}\right\}_{j=1}^{\infty} \subseteq\left\{x_{n}\right\}_{n=1}^{\infty}$.

(b) $X$ is said to have the Banach-Saks property if every weakly null sequence in $X$ has a subsequence which is a Banach-Saks sequence.

We remark that the classical formulation of the Banach-Saks property requires that each bounded sequence contain a Cesàro summable subsequence, and any Banach space enjoying this property is necessarily reflexive. See, for example, [Di]. In reflexive spaces, the classical Banach-Saks property is easily seen to be equivalent to the (so-called) weak Banach-Saks property which requires that each weakly null sequence should contain a Cesàro summable subsequence. That the apparent strengthening of the weak Banach-Saks property given in the preceding Definition 2.1(b) is, in fact, equivalent to the weak Banach-Saks property is due to Erdős and Magidor [EM]. See also [FS] and [Ro].

Throughout this paper, unless stated otherwise, we shall always assume that $E$ is a separable symmetric Banach function space on $\mathbb{R}^{+}$, that $(\mathcal{M}, \tau)$ is a semifinite von Neumann algebra and that $E(\mathcal{M}, \tau)$ is the Banach space of $\tau$-measurable operators associated with $(\mathcal{M}, \tau)$.

Proposition 2.2. If $E^{\times} \subseteq L_{0}[0, \infty)$ and if $a_{n}, y \in E(\mathcal{M}, \tau)$ satisfy $a_{n} \preccurlyeq y, n \in \mathbb{N}$, and $a_{n} \rightarrow 0$ for the measure topology, then $\left\|a_{n}\right\|_{E(\mathcal{M}, \tau)} \rightarrow 0$.

Proof. Without loss of generality, it may be assumed that $a_{n}^{*}=a_{n}$ for all $n \in \mathbb{N}$. Let $K$ be the unit ball of $E^{\times}(\mathcal{M}, \tau)=E(\mathcal{M}, \tau)^{*}$. Since $K$ is $\sigma\left(E(\mathcal{M}, \tau)^{\times}, E(\mathcal{M}, \tau)\right)$-sequentially compact, and using the assumptions that $E$ is separable and that $E^{\times} \subseteq L_{0}[0, \infty)$, it follows from [DScS, Theorem 5.4(i) $\Rightarrow$ (ii) and Proposition 2.2(iv) $\Rightarrow$ (vi)] that

$$
\left\|a_{n}\right\|_{E(\mathcal{M}, \tau)}=\sup \left\{\int_{[0, \infty)} \mu\left(a_{n}\right) \mu(y) d t: y \in K\right\} \rightarrow 0
$$

as $n \rightarrow \infty$. This suffices to prove the proposition.

In what follows, it will be convenient to denote by $\|\cdot\|_{+}$the norm on the space $L_{1}(\mathcal{M}, \tau)+\mathcal{M}$ so that

$$
\|x\|_{+}=\int_{0}^{1} \mu_{s}(x) d s, \quad x \in L_{1}(\mathcal{M}, \tau)+\mathcal{M} .
$$

As noted in [DSS], the separable space $L_{0}[0, \infty)$ fails to have the BanachSaks property. However, as the following proposition shows, equimeasurable 
sequences in $L_{0}(\mathcal{M}, \tau)$ contain subsequences with convergent Cesàro averages for all semifinite $(\mathcal{M}, \tau)$.

Proposition 2.3. If $\left\{x_{n}\right\}_{n=1}^{\infty} \subseteq L_{0}(\mathcal{M}, \tau)$ and if $\mu\left(x_{n}\right)=\mu\left(x_{1}\right), n \in \mathbb{N}$, then there exists a subsequence $\left\{x_{n(k)}\right\}_{k=1}^{\infty} \subseteq\left\{x_{n}\right\}_{n=1}^{\infty}$ and $x \in L_{0}(\mathcal{M}, \tau)$ such that

$$
\left\|x-\frac{1}{N} \sum_{k=1}^{N} x_{m(k)}\right\|_{+} \underset{N}{\rightarrow} 0
$$

for all further subsequences $\left\{x_{m(k)}\right\}_{k=1}^{\infty} \subset\left\{x_{n(k)}\right\}_{k=1}^{\infty}$.

Proof. For each $i \in \mathbb{N}$, let

$$
I_{i}=\left[\frac{1}{i}, \frac{1}{i-1}\right) \cup[i-1, i) .
$$

For each $n \geq 1$, we let $\mathcal{M}_{n}=\mathcal{M}_{\left|x_{n}\right|}, M_{n}=M_{\left|x_{n}\right|}, J_{n}=J_{\left|x_{n}\right|}$ be as given by Proposition 1.1 and let $\mathcal{E}_{n}$ be the conditional expectation of $\left(L_{1}+L_{\infty}\right)\left(\mathbb{R}^{+}\right)$ onto $\left(L_{1}+L_{\infty}\right)\left(M_{n}\right)$. Since $\mu\left(x_{n}\right)=\mu\left(x_{1}\right), n \in \mathbb{N}$, it follows that $M_{n}=$ $M_{1}:=M$ for all $n \in \mathbb{N}$, and so also $\mathcal{E}_{n}=\mathcal{E}_{1}:=\mathcal{E}$. Let $x_{n}=u_{n}\left|x_{n}\right|$ be the polar decomposition of $x_{n}, n=1,2, \ldots$ We set

$$
z_{n}^{(i)}=J_{n}\left(\mathcal{E}\left(\chi_{I_{i}}\right)\right), \quad i \geq 2, n \geq 1,
$$

and, using Proposition 1.1, observe that

$$
\left|x_{n}\right| z_{n}^{(i)}=J_{n}\left(\mu\left(x_{1}\right)\right) J_{n}\left(\mathcal{E}\left(\chi_{I_{i}}\right)\right)=J_{n}\left(\mathcal{E}\left(\mu\left(x_{1}\right) \chi_{I_{i}}\right)\right)
$$

so that

$$
\mu\left(\left|x_{n}\right| z_{n}^{(i)}\right)=\mu\left(J_{n}\left(\mathcal{E}\left(\mu\left(x_{1}\right) \chi_{I_{i}}\right)\right)=\mu\left(\mu\left(x_{1}\right) \chi_{I_{i}}\right), \quad i \geq 2, n \geq 1 .\right.
$$

Since

$$
\begin{aligned}
\sup _{n}\left\|x_{n} z_{n}^{(i)}\right\|_{\left.L_{2}(\mathcal{M}, \tau)\right)} & =\sup _{n}\left\|u_{n}\left|x_{n}\right| z_{n}^{(i)}\right\|_{\left.L_{2}(\mathcal{M}, \tau)\right)} \leq \sup _{n}\left\|\left|x_{n}\right| z_{n}^{(i)}\right\|_{\left.L_{2}(\mathcal{M}, \tau)\right)} \\
& \leq\left\|\mu\left(x_{1}\right) \chi_{I_{i}}\right\|_{L_{2}[0, \infty)}
\end{aligned}
$$

and since $L_{2}(\mathcal{M}, \tau)$ has the Banach-Saks property, it follows from a diagonal argument that there exists a subsequence $\left\{x_{n(j)}\right\}_{j=1}^{\infty} \subseteq\left\{x_{n}\right\}_{n=1}^{\infty}$ such that, for all $2 \leq i \in \mathbb{N}$, there exists $x^{(i)} \in L_{2}(\mathcal{M}, \tau)$ such that

$$
\lim _{N \rightarrow \infty} \frac{1}{N} \sum_{j=1}^{N} x_{n(j)} z_{n(j)}^{(i)}=x^{(i)}
$$

holds in $L_{2}(\mathcal{M}, \tau)$, and hence also in $L_{1}(\mathcal{M}, \tau)+\mathcal{M}$, for all further subsequences $\left\{x_{m(j)}\right\}_{j=1}^{\infty} \subset\left\{x_{n(k)}\right\}_{k=1}^{\infty}$. We let $\left\{x_{m(j)}\right\}_{j=1}^{\infty} \subset\left\{x_{n(k)}\right\}_{k=1}^{\infty}$ be a fixed subsequence and set

$$
w_{N}:=\frac{1}{N} \sum_{k=1}^{N} x_{m(k)}, \quad N=1,2, \ldots,
$$


and let $\varepsilon>0$ be given. We observe that, for every $m=1,2, \ldots$, and $M, N \in \mathbb{N}$,

$$
\begin{aligned}
\left\|w_{N}-w_{M}\right\|_{+} \leq & \left\|\frac{1}{N} \sum_{k=1}^{N}\left(x_{m(k)}-\sum_{i=1}^{m} x_{m(k)} z_{m(k)}^{(i)}\right)\right\|_{+} \\
& +\left\|\frac{1}{M} \sum_{k=1}^{M}\left(x_{m(k)}-\sum_{i=1}^{m} x_{m(k)} z_{m(k)}^{(i)}\right)\right\|_{+} \\
& +\left\|\sum_{i=1}^{m} x^{(i)}-\frac{1}{N} \sum_{k=1}^{N} \sum_{i=1}^{m} x_{m(k)} z_{m(k)}^{(i)}\right\|_{+} \\
& +\left\|\sum_{i=1}^{m} x^{(i)}-\frac{1}{M} \sum_{k=1}^{M} \sum_{i=1}^{m} x_{m(k)} z_{m(k)}^{(i)}\right\|_{+}
\end{aligned}
$$

Choose $m \in \mathbb{N}$ such that

$$
1 / m<\varepsilon, \quad \mu_{m}\left(x_{1}\right)<\varepsilon, \quad \int_{0}^{1 / m} \mu_{s}\left(x_{1}\right) d s<\varepsilon .
$$

Noting that

$$
\begin{aligned}
\mu\left(x_{m(k)}-\sum_{i=1}^{m} x_{m(k)} z_{m(k)}^{(i)}\right) & =\mu\left(u_{k}\left|x_{m(k)}\right| \sum_{i=m+1}^{\infty} z_{m(k)}^{(i)}\right) \\
& \leq \mu\left(\left|x_{m(k)}\right| \sum_{i=m+1}^{\infty} z_{m(k)}^{(i)}\right) \\
& =\mu\left(J_{m(k)} \mathcal{E}\left(\mu\left(x_{1}\right) \sum_{i=m+1}^{\infty} \chi_{I_{i}}\right)\right) \\
& =\mu\left(\mathcal{E}\left(\mu\left(x_{1}\right) \sum_{i=m+1}^{\infty} \chi_{I_{i}}\right)\right) \ll \mu\left(\mu\left(x_{1}\right) \sum_{i=m+1}^{\infty} \chi_{I_{i}}\right),
\end{aligned}
$$

and that

$$
\mu_{t}\left(\mu\left(x_{1}\right) \sum_{i=m+1}^{\infty} \chi_{I_{i}}\right)= \begin{cases}\mu_{t}\left(x_{1}\right) & \text { if } 0<t<1 / m \\ \mu_{t+m}\left(x_{1}\right) & \text { if } t \geq 1 / m\end{cases}
$$

it follows that

$$
\begin{aligned}
\left\|x_{m(k)}-\sum_{i=1}^{m} x_{m(k)} z_{m(k)}^{(i)}\right\|_{+} & =\int_{0}^{1} \mu_{s}\left(x_{m(k)}-\sum_{i=1}^{m} x_{m(k)} z_{m(k)}^{(i)}\right) d s \\
& \leq \int_{0}^{1 / m} \mu_{s}\left(x_{1}\right) d s+\int_{1 / m}^{1} \varepsilon d s \leq 2 \varepsilon .
\end{aligned}
$$


Since

$$
\frac{1}{N} \sum_{k=1}^{N}\left(x_{m(k)}-\sum_{i=1}^{m} x_{m(k)} z_{m(k)}^{(i)}\right) \ll \frac{1}{N} \sum_{k=1}^{N} \mu\left(x_{m(k)}-\sum_{i=1}^{m} x_{m(k)} z_{m(k)}^{(i)}\right),
$$

it follows further that

$$
\left\|\frac{1}{N} \sum_{k=1}^{N}\left(x_{m(k)}-\sum_{i=1}^{m} x_{m(k)} z_{m(k)}^{(i)}\right)\right\|_{+} \leq 2 \varepsilon,
$$

and similarly

$$
\left\|\frac{1}{M} \sum_{k=1}^{M}\left(x_{m(k)}-\sum_{i=1}^{m} x_{m(k)} \chi z_{m(k)}^{(i)}\right)\right\|_{+} \leq 2 \varepsilon
$$

for all $M, N \in \mathbb{N}$. Now observing that

$$
\left\|\sum_{i=1}^{m} x^{(i)}-\frac{1}{N} \sum_{k=1}^{N} \sum_{i=1}^{m} x_{m(k)} z_{m(k)}^{(i)}\right\|_{+}=\left\|\sum_{i=1}^{m}\left(x^{(i)}-\frac{1}{N} \sum_{k=1}^{N} x_{m(k)} z_{m(k)}^{(i)}\right)\right\|_{+}
$$

together with the same equality with $N$ replaced by $M$, and using (2.5), it follows that there exists $N_{0} \in \mathbb{N}$ such that

$$
\left\|\sum_{i=1}^{m} x^{(i)}-\frac{1}{N} \sum_{k=1}^{N} \sum_{i=1}^{m} x_{m(k)} z_{m(k)}^{(i)}\right\|_{+}+\left\|\sum_{i=1}^{m} x^{(i)}-\frac{1}{M} \sum_{k=1}^{M} \sum_{i=1}^{m} x_{m(k)} z_{m(k)}^{(i)}\right\|_{+} \leq \varepsilon,
$$

for all $M, N \geq N_{0}$. We obtain

$$
\left\|w_{N}-w_{M}\right\|_{+} \leq 5 \varepsilon
$$

for all $M, N \geq N_{0}$. Consequently, there exists $x \in L_{1}(\mathcal{M}, \tau)+\mathcal{M}$ such that

$$
\frac{1}{N} \sum_{k=1}^{N} x_{m(k)} \rightarrow x
$$

as $N \rightarrow \infty$. To show that $x$ is independent of the subsequence $\left\{x_{m(k)}\right\}_{k=1}^{\infty}$, let $\varepsilon>0$ be given and suppose that $m$ satisfies (2.6). By (2.7),

$$
\begin{aligned}
\left\|\frac{1}{N} \sum_{k=1}^{N} x_{m(k)}-\sum_{i=1}^{m}\left(\frac{1}{N} \sum_{k=1}^{N} x_{m(k)} z_{m(k)}^{(i)}\right)\right\|_{+} \\
=\left\|\frac{1}{N} \sum_{k=1}^{N}\left(x_{m(k)}-\sum_{i=1}^{m} x_{m(k)} z_{m(k)}^{(i)}\right)\right\|_{+} \leq 2 \varepsilon
\end{aligned}
$$

for all $N \in \mathbb{N}$. Letting $N \rightarrow \infty$, we obtain

$$
\left\|x-\sum_{i=1}^{m} x^{(i)}\right\|_{+}<2 \varepsilon
$$


for all sufficiently large $m \in \mathbb{N}$. This shows that the equality $x=\sum_{i=1}^{\infty} x^{(i)}$ holds in $L_{1}(\mathcal{M}, \tau)+\mathcal{M}$. Since $L_{0}(\mathcal{M}, \tau)$ is closed in $L_{1}(\mathcal{M}, \tau)+\mathcal{M}$, it follows that $x \in L_{0}(\mathcal{M}, \tau)$, and this suffices to complete the proof.

If $x \in \widetilde{\mathcal{M}}$, then the right and left support projections of $x$ are denoted by $r(x), l(x)$ respectively. Note that if $x=u|x|$ is the polar decomposition, then $u^{*} u=r(x)$ and $u u^{*}=l(x)$ (see, for example, [Ta]). The family $\left\{x_{i}\right\}_{i \in \mathcal{I}} \subset \widetilde{\mathcal{M}}$ is said to be right (respectively, left) disjointly supported if $r\left(x_{i}\right) r\left(x_{j}\right)=0$ (respectively, $l\left(x_{i}\right) l\left(y_{j}\right)=0$ ) for all $i, j \in \mathcal{I}, i \neq j$.

We shall need the following assertion that sequences converging to zero for the measure topology contain subsequences that are approximately both right and left disjointly supported. The lemma which follows is proved in [CDS, Theorem 2.5] and, in the case that $E=L_{p}[0,1), p \geq 1$, is due to Kadec and Pełczyński [KP].

Lemma 2.4. If $\left\{x_{n}\right\}_{n=1}^{\infty} \subseteq E(\mathcal{M}, \tau)$ converges to zero in the measure topology, then there exists a subsequence $\left\{y_{n}\right\}_{n=1}^{\infty} \subseteq\left\{x_{n}\right\}_{n=1}^{\infty}$ and sequences $\left\{p_{n}\right\},\left\{q_{n}\right\}$ of mutually orthogonal projections in $\mathcal{M}$ such that

$$
\left\|y_{n}-p_{n} y_{n} q_{n}\right\|_{E(\mathcal{M}, \tau)} \rightarrow 0 .
$$

The preceding lemma implies immediately the following non-commutative extension of a variant of the Kadec-Pełczyński lemma as given in [HLR, Corollary 3.6].

Corollary 2.5. If $Y \subseteq E(\mathcal{M}, \tau)$ is a closed subspace, then one of the following statements holds:

(i) The norm topology of $E(\mathcal{M}, \tau)$ coincides on $Y$ with the measure topology.

(ii) There is a sequence $\left\{y_{n}\right\}_{n=1}^{\infty}$ in the unit sphere of $Y$ and a twosided disjointly supported sequence $\left\{d_{n}\right\}_{n=1}^{\infty} \subseteq E(\mathcal{M}, \tau)$ such that $\left\|y_{n}-d_{n}\right\|_{E(\mathcal{M}, \tau)} \rightarrow 0$.

The following observation is an immediate consequence of a well known characterisation of weakly compact sets in the predual of a von Neumann algebra due to C. A. Akemann.

Lemma 2.6. If $\left\{x_{n}\right\}_{n=1}^{\infty} \subseteq E(\mathcal{M}, \tau)$ is weakly convergent and is left disjointly supported, then $\left\{x_{n}\right\}_{n=1}^{\infty}$ is weakly null.

Proof. Suppose that $x \in E(\mathcal{M}, \tau)$ satisfies $x_{n} \rightarrow x$ weakly in $E(\mathcal{M}, \tau)$. If $z \in E(\mathcal{M}, \tau)^{*}=E(\mathcal{M}, \tau)^{\times}$, then $x_{n} z \rightarrow x z$ weakly in $L_{1}(\mathcal{M}, \tau)$. If $e_{n}$ is the left support of $x_{n}, n \geq 1$, then by assumption the sequence $\left\{e_{n}\right\}_{n=1}^{\infty}$ is pairwise disjoint, and consequently, from the well known Akemann criterion (see, for example, [RX, Theorem $4.14(\mathrm{i}) \Leftrightarrow(\mathrm{ii})]$ ), it follows that

$$
\left|\tau\left(x_{n} z\right)\right|=\left|\tau\left(e_{n} x_{n} z\right)\right| \leq \sup _{k}\left|\tau\left(e_{n} x_{k} z\right)\right| \rightarrow 0 .
$$


The subsequence splitting principle which follows shows that in separable spaces with the Fatou property, each bounded sequence contains a subsequence which is a perturbation of the sum of an equimeasurable sequence and a sequence which is left and right disjointly supported.

Proposition 2.7. Suppose that $E$ has the Fatou property, and suppose that $(\mathcal{M}, \tau)$ is non-atomic. Let $\left\{x_{n}\right\}_{n=1}^{\infty} \subseteq E(\mathcal{M}, \tau)$ be a sequence of selfadjoint elements with

$$
\sup _{n \in \mathbb{N}}\left\|x_{n}\right\|_{E(\mathcal{M}, \tau)}=C<\infty .
$$

(i) There exists a subsequence $\left\{x_{n}^{\prime}\right\}_{n=1}^{\infty} \subseteq\left\{x_{n}\right\}_{n=1}^{\infty}$ which admits the splitting

$$
x_{n}^{\prime}=y_{n}+z_{n}+d_{n}, \quad n \geq 1,
$$

where $\left\{y_{n}\right\}_{n=1}^{\infty},\left\{z_{n}\right\}_{n=1}^{\infty},\left\{d_{n}\right\}_{n=1}^{\infty} \subseteq E(\mathcal{M}, \tau)$ are bounded sequences satisfying

(a) the sequence $\left\{y_{n}\right\}_{n=1}^{\infty}$ is equimeasurable, that is, $\mu\left(y_{1}\right)=\mu\left(y_{n}\right)$ for all $n \in \mathbb{N}$, and $\left\|y_{1}\right\|_{E(\mathcal{M}, \tau)} \leq C$

(b) $z_{n} \rightarrow 0$ for the measure topology and there exist sequences $\left\{p_{n}\right\}$, $\left\{q_{n}\right\}$ of mutually orthogonal projections in $\mathcal{M}$ such that $z_{n}=$ $p_{n} z_{n} q_{n}$ for all $n \in \mathbb{N}$ and $\sup _{n \in \mathbb{N}}\left\|z_{n}\right\|_{E(\mathcal{M}, \tau)} \leq 2 C$;

(c) $\left\|d_{n}\right\|_{E} \rightarrow 0$.

(ii) If , in addition, $E^{\times} \subseteq L_{0}[0, \infty)$ and the sequence $\left\{x_{n}\right\}_{n=1}^{\infty}$ is weakly null, then the sequences $\left\{y_{n}\right\}_{n=1}^{\infty},\left\{z_{n}\right\}_{n=1}^{\infty}$ from (2.8) may be chosen to be weakly null as well.

Proof. (i) It follows from the inequalities

$$
\sup _{n} \mu_{t}\left(x_{n}\right) \leq C /\left\|\chi_{[0, t)}\right\|_{E}, \quad t>0,
$$

that the sequence $\left\{\mu\left(x_{n}\right)\right\}_{n=1}^{\infty}$ is uniformly bounded on every interval of the form $[a, b]$ for all $0<a<b<\infty$. The Helly selection theorem and a diagonal argument show that there exists a subsequence $\left\{x_{n}^{\prime}\right\}_{n=1}^{\infty} \subseteq\left\{x_{n}\right\}_{n=1}^{\infty}$ and a right-continuous, non-increasing function $f:(0, \infty) \rightarrow[0, \infty)$ such that $\mu\left(x_{n}^{\prime}\right) \rightarrow f$ almost everywhere on $\mathbb{R}^{+}$. Since $E$ has the Fatou property, it follows that $f \in E$ and that $\|f\|_{E} \leq C$.

We set

$$
a_{n}(t):=\mu_{t}\left(x_{n}^{\prime}\right)-f(t), \quad \forall t>0, \forall n \in \mathbb{N} .
$$

Since $E$ is separable, $\lim _{t \rightarrow \infty} f(t)=\lim _{t \rightarrow \infty} \mu_{t}\left(x_{n}^{\prime}\right)=0$, and since $f, \mu\left(x_{n}\right)$ are non-increasing for all $n \in \mathbb{N}$, it follows that $a_{n} \rightarrow 0$ in measure.

Proposition 1.1 implies that there exists a rearrangement-preserving mapping $J_{n}: E \rightarrow E(\mathcal{M}, \tau)$ such that $J_{n}\left(\mu\left(x_{n}^{\prime}\right)\right)=\left|x_{n}^{\prime}\right|$ for all $n \in \mathbb{N}$. 
We let $x_{n}^{\prime}=u_{n}\left|x_{n}^{\prime}\right|$ be the polar decomposition and note that since $x_{n}$ is self-adjoint, $u_{n}$ may be taken to be unitary. We now set

$$
y_{n}=u_{n} J_{n}(f), \quad w_{n}=u_{n} J_{n}\left(a_{n}\right)=u_{n} J_{n}\left(\mu\left(x_{n}^{\prime}\right)-f\right), \quad n \in \mathbb{N} .
$$

It follows immediately that

$$
x_{n}^{\prime}=u_{n}\left|x_{n}^{\prime}\right|=u_{n} J_{n}\left(\mu\left(x_{n}^{\prime}\right)\right)=y_{n}+w_{n}
$$

and that

$$
\mu\left(y_{n}\right)=f, \quad n \in \mathbb{N} .
$$

In particular,

$$
\left\|y_{n}\right\|_{E(\mathcal{M}, \tau)}=\|f\|_{E} \leq C, \quad\left\|w_{n}\right\|_{E(\mathcal{M}, \tau)} \leq 2 C
$$

for all $n \in \mathbb{N}$. Since $a_{n} \rightarrow 0$ in measure, we also have $w_{n}=u_{n} J_{n}\left(a_{n}\right) \rightarrow 0$ in measure. By the separability of $E$, Lemma 2.4 shows that, by passing to a subsequence if necessary and relabelling, there exist sequences $\left\{p_{n}\right\},\left\{q_{n}\right\}$ of mutually orthogonal projections in $\mathcal{M}$ such that $\left\|w_{n}-p_{n} w_{n} q_{n}\right\|_{E(\mathcal{M}, \tau)} \rightarrow 0$. Setting $z_{n}=p_{n} w_{n} q_{n}$ and $d_{n}=w_{n}-p_{n} w_{n} q_{n}$ for all $n \in \mathbb{N}$, it follows that $\left\|z_{n}\right\|_{E(\mathcal{M}, \tau)} \leq 2 C$ and $\left\|d_{n}\right\|_{E(\mathcal{M}, \tau)} \rightarrow 0$, as required.

(ii) From part (i), we may assume that the decomposition (2.8) holds for the sequence $\left\{x_{n}\right\}_{n=1}^{\infty}$. We set $f=\mu\left(y_{1}\right)$ and let

$$
\Omega(f):=\{x \in E(\mathcal{M}, \tau): \mu(x) \nVdash f\} .
$$

Using the assumption that $E^{\times} \subseteq L_{0}[0, \infty)$ and the separability of $E$, it follows from [DScS, Theorem 5.4] that $\Omega(f)$ is sequentially compact for the weak topology on $E(\mathcal{M}, \tau)$ induced by $E(\mathcal{M}, \tau)^{\times}$. Passing again to a subsequence if necessary, we may assume that $\left\{y_{n}\right\}_{n=1}^{\infty} \subseteq \Omega(f)$ is weakly convergent, and using the assumption that $\left\{x_{n}\right\}_{n=1}^{\infty}$ is weakly null, we may assume further that the two-sided disjoint sequence $\left\{z_{n}\right\}_{n=1}^{\infty}$ is weakly convergent. It now follows from Lemma 2.6 that $\left\{z_{n}\right\}_{n=1}^{\infty}$ is weakly null. This implies that $\left\{y_{n}\right\}_{n=1}^{\infty}$ is also weakly null, and completes the proof of the proposition.

In the commutative setting, the preceding proposition is given in [DSS, Proposition 3.2]. In the case that $\tau(\mathbf{1})<\infty$, part (i) of the preceding proposition was established in [Su, Lemma 1.1 and Proposition 2.2]. If we observe that each equimeasurable sequence in the space $L_{p}[0,1), 1 \leq p<\infty$, is $L_{p}$-equiintegrable (see Definition 3.3 below and the discussion following it), this implies the classical subsequence splitting principle of Kadec and Pełczyński $[\mathrm{KP}]$ which states that each bounded sequence in $L_{p}[0,1)$ contains a subsequence which is the sum of a sequence which is $L_{p}$-equiintegrable and a sequence which is disjointly supported. More recently, it has been shown by N. Randrianantoanina [Ran2] (see also [RX]) that if $E$ satisfies a non-trivial lower estimate then each bounded sequence in $E(\mathcal{M}, \tau)$ contains 
a subsequence which splits as the sum of an E-equiintegrable sequence and a sequence which is disjointly supported. In the case of finite trace, this result is implied by the present Proposition 2.7(i), but in the case that the trace is not finite, the subsequence splitting principles given by Proposition 2.7(i) and [Ran2] are quite distinct.

The theorem which follows is a non-commutative analogue of the well known theorem of Komlós [Ko]. For the special case that $\tau(\mathbf{1})<\infty$ and $E=L_{1}$, this result is proved in [Ran1] using somewhat different techniques.

Theorem 2.8. Suppose that $E$ has the Fatou property. If $\left\{x_{n}\right\}_{n=1}^{\infty}$ $\subseteq E(\mathcal{M}, \tau)$ satisfies $\sup \left\|x_{n}\right\|_{E(\mathcal{M}, \tau)}<\infty$, then there exists $y \in E(\mathcal{M}, \tau)$ and a subsequence $\left\{y_{n}\right\}_{n=1}^{\infty} \subseteq\left\{x_{n}\right\}_{n=1}^{\infty}$ such that for every further subsequence $\left\{y_{n(k)}\right\}_{k=1}^{\infty} \subseteq\left\{y_{n}\right\}_{n=1}^{\infty}$, the sequence of averages $\left\{\sum_{k=1}^{n} y_{n(k)} / n\right\}_{n=1}^{\infty}$ converges to $y$ in the measure topology.

Proof. It clearly suffices to assume that the sequence $\left\{x_{n}\right\}$ consists of self-adjoint elements. We assume first that $(\mathcal{M}, \tau)$ is non-atomic. By Proposition 2.7(i) and passing to a subsequence and relabelling if necessary, we may assume that $\mu\left(x_{n}\right)=\mu\left(x_{1}\right)$ for all $n \geq 1$. By Proposition 2.3, using the fact that $E$ is separable, there exists $y \in L_{0}(\mathcal{M}, \tau)$ and a subsequence $\left\{y_{n}\right\}_{n=1}^{\infty} \subseteq\left\{x_{n}\right\}_{n=1}^{\infty}$ such that the sequence $\left\{\sum_{k=1}^{n} y_{n(k)} / n\right\}_{n=1}^{\infty}$ converges to $y$ in the norm of $L_{1}(\mathcal{M}, \tau)+\mathcal{M}$, and hence for the measure topology, for all further subsequences $\left\{y_{n(k)}\right\}_{k=1}^{\infty} \subseteq\left\{y_{n}\right\}_{n=1}^{\infty}$. Since $\sup \left\|x_{n}\right\|_{E(\mathcal{M}, \tau)}<\infty$, and since $E$ has the Fatou property, it follows from [DDP3, Proposition 5.14] that $y \in E(\mathcal{M}, \tau)$.

In the general case, let

$$
\mathcal{N}:=\mathcal{M} \otimes L_{\infty}(0,1), \quad \tau_{\mathcal{N}}:=\tau \otimes m .
$$

Clearly $\mathcal{M} \otimes \chi_{[0,1]}$ is a proper von Neumann subalgebra of $\left(\mathcal{N}, \tau_{\mathcal{N}}\right)$. We may obviously identify the former subalgebra with $\mathcal{M}$ by considering the mapping

$$
\phi: \widetilde{\mathcal{M}} \rightarrow \widetilde{\mathcal{N}}, \quad \phi(x):=x \otimes \chi_{[0,1]} .
$$

For the necessary details, we refer to $[\mathrm{FK}]$, [CS]. By the first part of the proof, there exists a subsequence $\left\{y_{n}\right\}_{n=1}^{\infty} \subseteq\left\{x_{n}\right\}_{n=1}^{\infty}$ and there exists $z \in$ $E\left(\mathcal{N}, \tau_{\mathcal{N}}\right)$ such that the sequence $\left\{\sum_{k=1}^{n} \phi\left(y_{n(k)}\right) / n\right\}_{n=1}^{\infty}$ converges to $z$ for the measure topology on $\tilde{\mathcal{N}}$. It follows that the sequence $\left\{\sum_{k=1}^{n} y_{n(k)} / n\right\}_{n=1}^{\infty}$ is Cauchy for the measure topology on $\widetilde{\mathcal{M}}$ and hence converges to some element $y \in \widetilde{\mathcal{M}}$ for the measure topology on $\widetilde{\mathcal{M}}$. Therefore we readily see that $z=\phi(y)$, and this implies further that $y \in E(\mathcal{M}, \tau)$. This suffices to complete the proof of the theorem.

The following lemma is given in [PSW, Lemma 5.3]. 
Lemma 2.9. Let $\left\{x_{n}\right\}_{n=1}^{\infty}$ be a sequence in $L_{1}(\mathcal{M}, \tau)+\mathcal{M}$. If $x \in \widetilde{\mathcal{M}}$ and $y \in L_{1}(\mathcal{M}, \tau)+\mathcal{M}$ are such that $x_{n} \rightarrow x$ for the measure topology and $x_{n} \rightarrow y$ for the weak topology on $L_{1}(\mathcal{M}, \tau)+\mathcal{M}$ induced by $L_{1}(\mathcal{M}, \tau) \cap \mathcal{M}$, then $x=y$.

Definition 2.10. A sequence $\left\{x_{n}\right\}_{n=1}^{\infty} \subseteq E(\mathcal{M}, \tau)$ will be said to be E-equiintegrable at 0 if

$$
\lim _{\delta \rightarrow 0} \sup _{n \in \mathbb{N}}\left\|\mu\left(x_{n}\right) \chi_{[0, \delta)}\right\|_{E}=0 .
$$

Proposition 2.11. Suppose that $(\mathcal{M}, \tau)$ is non-atomic. If $\left\{x_{n}\right\}_{n=1}^{\infty} \subseteq$ $E(\mathcal{M}, \tau)$, then the following statements are equivalent:

(i) $\left\{x_{n}\right\}_{n=1}^{\infty}$ is E-equiintegrable at 0 .

(ii) $\lim _{e \in \mathcal{P}, \tau(e) \rightarrow 0} \sup _{n \in \mathbb{N}}\left\|x_{n} e\right\|_{E(\mathcal{M}, \tau)}=0$.

(iii) $\lim _{e \in \mathcal{P}, \tau(e) \rightarrow 0} \sup _{n \in \mathbb{N}}\left\|e x_{n}\right\|_{E(\mathcal{M}, \tau)}=0$.

(iv) $\lim _{e \in \mathcal{P}, \tau(e) \rightarrow 0} \sup _{n \in \mathbb{N}}\left\|e x_{n} e\right\|_{E(\mathcal{M}, \tau)}=0$.

Proof. The implications (i) $\Rightarrow($ ii $) \Rightarrow($ iv), (i) $\Rightarrow($ iii $) \Rightarrow($ iv) follow immediately from the submajorisations

$$
\mu(e x e) \leq \mu(x e), \quad \mu(e x) \prec \mu(x) \chi_{[0, \tau(e))}
$$

for each $e \in \mathcal{P}$ and $x \in E(\mathcal{M}, \tau)$ (see [FK, Theorem 4.2(iii)]). To show that (iv) $\Rightarrow(\mathrm{i})$, it suffices to assume that $x_{n}^{*}=x_{n}$ for all $n \in \mathbb{N}$. If $x \in E(\mathcal{M})$ is self-adjoint and $\delta>0$, then it follows from the fact that $(\mathcal{M}, \tau)$ is non-atomic and Proposition 1.1 that there exists $e \in \mathcal{P}$ commuting with $x$ such that $\tau(e)=\delta$ and such that

$$
\mu(x) \chi_{[0, \delta)}=\mu(x e)=\mu(e x e) .
$$

The implication (iv) $\Rightarrow($ i) now follows readily from this observation and the details are omitted.

We shall need the following variant of Proposition 2.7.

Proposition 2.12. Suppose that $E$ has the Fatou property and suppose that $(\mathcal{M}, \tau)$ is non-atomic. Let $\left\{x_{n}\right\}_{n=1}^{\infty}$ be a sequence of self-adjoint elements with

$$
\sup _{n \in \mathbb{N}}\left\|x_{n}\right\|_{E(\mathcal{M}, \tau)}<\infty
$$

(i) There exists a subsequence $\left\{y_{n}\right\}_{n=1}^{\infty} \subseteq\left\{x_{n}\right\}_{n=1}^{\infty}$ and there exist sequences $\left\{v_{n}\right\}_{n=1}^{\infty},\left\{w_{n}\right\}_{n=1}^{\infty} \subseteq E(\mathcal{M}, \tau)$ such that $y_{n}=v_{n}+w_{n}$, $\left\{w_{n}\right\}_{n=1}^{\infty}$ is E-equiintegrable at 0 and $\left\{v_{n}\right\}_{n=1}^{\infty}$ converges to 0 in measure.

(ii) If $\left\{x_{n}\right\}_{n=1}^{\infty}$ is weakly null, then each of the sequences $\left\{v_{n}\right\}_{n=1}^{\infty}$, $\left\{w_{n}\right\}_{n=1}^{\infty}$ may be taken to be weakly null. 
Proof. Passing to a subsequence if necessary, a standard argument based on Helly's theorem as in the proof of Proposition 2.7 yields the existence of a non-increasing function $f$ on $(0, \infty)$ such that $\mu\left(x_{n}\right) \rightarrow f$ almost everywhere. Since $E$ has the Fatou property, we infer that $f \in E$. Observe that for all $0<a<b<\infty$, it follows from the bounded convergence theorem that $\left\|\mu\left(x_{n}\right) \chi_{[a, b)}-f \chi_{[a, b)}\right\|_{E} \rightarrow 0$. Suppose that $\delta_{n}, \varepsilon_{n} \downarrow 0$ with $\sum_{n=1}^{\infty} \delta_{n}<\infty$ and $0<\delta_{n}<1$ for all $n \in \mathbb{N}$. Passing to subsequences, we may assume that

$$
\left\|\mu\left(x_{n}\right) \chi_{\left[\delta_{n}, 1\right)}-f \chi_{\left[\delta_{n}, 1\right)}\right\|_{E}<\varepsilon_{n}, \quad n \in \mathbb{N} .
$$

We let $x_{n}=u_{n}\left|x_{n}\right|$ be the polar decomposition, with $u_{n}$ taken to be unitary by the self-adjointness of $x_{n}$, for all $n \in \mathbb{N}$. Again by Proposition 1.1, there exists a rearrangement-preserving mapping $J_{n}: E \rightarrow E(\mathcal{M}, \tau)$ such that $J_{n}\left(\mu\left(x_{n}\right)\right)=\left|x_{n}\right|$ for all $n \in \mathbb{N}$. We now set

$$
v_{n}=u_{n} J_{n}\left(\mu\left(x_{n}\right) \chi_{\left[0, \delta_{n}\right)}\right), \quad w_{n}=u_{n} J_{n}\left(\mu\left(x_{n}\right) \chi_{\left[\delta_{n}, \infty\right)}\right), \quad n \in \mathbb{N} .
$$

It is clear that $x_{n}=v_{n}+w_{n}, n \in \mathbb{N}$, and that $v_{n} \rightarrow 0$ in measure. To show that the sequence $\left\{w_{n}\right\}$ is $E$-equiintegrable at 0 , let $\alpha_{n} \downarrow 0$. Without loss of generality we may assume that $0<\alpha_{n}+\delta_{n}<1$ and that $\left\|f \chi_{\left[0, \alpha_{n}\right)}\right\|_{E}<\varepsilon_{n}$ for all $n \in \mathbb{N}$. Observe that

$$
\begin{aligned}
\left\|\mu\left(w_{n}\right) \chi_{\left[0, \alpha_{n}\right)}\right\|_{E} & =\left\|\mu\left(\mu\left(x_{n}\right) \chi_{\left[\delta_{n}, \infty\right)}\right) \chi_{\left[0, \alpha_{n}\right)}\right\|_{E} \\
& \leq\left\|\mu\left(x_{n}\right) \chi_{\left[\delta_{n}, \delta_{n}+\alpha_{n}\right)}-f \chi_{\left[\delta_{n}, \delta_{n}+\alpha_{n}\right)}\right\|_{E}+\left\|f \chi_{\left[\delta_{n}, \delta_{n}+\alpha_{n}\right)}\right\|_{E} \\
& \leq\left\|\mu\left(x_{n}\right) \chi_{\left[\delta_{n}, 1\right)}-f \chi_{\left[\delta_{n}, 1\right)}\right\|_{E}+\left\|f \chi_{\left[0, \alpha_{n}\right)}\right\|_{E} \leq 2 \varepsilon_{n}
\end{aligned}
$$

for all $n \in \mathbb{N}$. From this, it follows readily that the sequence $\left\{w_{n}\right\}_{n=1}^{\infty}$ is $E$-equintegrable at 0.

Suppose now that the sequence $\left\{x_{n}\right\}_{n=1}^{\infty}$ is weakly null. It follows from [DScS, Theorem 5.2] that $\left\{\mu\left(x_{n}\right)\right\}_{n=1}^{\infty} \subseteq E$ is relatively weakly compact. By [Fr, 82G], it follows that $\left\{\mu\left(v_{n}\right)\right\}_{n=1}^{\infty}=\left\{\mu\left(x_{n}\right) \chi_{\left[0, \delta_{n}\right)}\right\}_{n=1}^{\infty}$ is relatively weakly compact. Since $\mu\left(v_{n}\right) \rightarrow 0$ in measure, Lemma 2.9 shows that $\left\{\mu\left(v_{n}\right)\right\}_{n=1}^{\infty}$ is weakly null. For all $n \in \mathbb{N}$, we denote by $e_{n}^{\prime}, e_{n}^{\prime \prime}$, respectively, the left and right support projections of $v_{n}$, and set

$$
e=\sup _{n \in \mathbb{N}} e_{n}^{\prime} \vee e_{n}^{\prime \prime}
$$

It follows that

$$
0 \leq \tau(e) \leq \sum_{n=1}^{\infty}\left(\tau\left(e_{n}^{\prime}\right)+\tau\left(e_{n}^{\prime \prime}\right)\right) \leq 2 \sum_{n=1}^{\infty} \delta_{n}<\infty .
$$

Since $\left\{v_{n}\right\}_{n=1}^{\infty} \subseteq e \mathcal{M} e$, we now deduce from [DScS, Theorem 5.4] that the sequence $\left\{v_{n}\right\}_{n=1}^{\infty}$ is relatively weakly compact in $E(e \mathcal{M} e, \tau(e \cdot e))$ and hence relatively weakly compact in $E(\mathcal{M}, \tau)$. Since $v_{n} \rightarrow 0$ in measure, it again follows from Lemma 2.9 that any weak cluster point of $\left\{v_{n}\right\}_{n=1}^{\infty}$ is necessar- 
ily 0 . From this, it follows that $\left\{v_{n}\right\}_{n=1}^{\infty}$ (and hence also $\left\{w_{n}\right\}_{n=1}^{\infty}$ ) is weakly null.

Theorem 2.13. If $E$ has the Fatou property and if $\mathcal{M}$ is non-atomic, then the following conditions are equivalent:

(i) $E(\mathcal{M}, \tau)$ has the Banach-Saks property.

(ii) Each weakly null two-sided disjointly supported sequence in $E(\mathcal{M}, \tau)$ contains a Banach-Saks subsequence.

Proof. We only need to prove that (ii) implies (i). Let $\left\{x_{n}\right\}_{n=1}^{\infty} \subseteq E(\mathcal{M}, \tau)$ be a weakly null sequence. We assume first that $E^{\times} \subseteq L_{0}[0, \infty)$. By Proposition 2.7(ii), and passing to a subsequence if necessary, we may assume that there exist weakly null sequences $\left\{y_{n}\right\}_{n=1}^{\infty} \subseteq E(\mathcal{M}, \tau)$ and $\left\{z_{n}\right\}_{n=1}^{\infty} \subseteq$ $E(\mathcal{M}, \tau)$ and a null sequence $\left\{d_{n}\right\}_{n=1}^{\infty} \subseteq E(\mathcal{M}, \tau)$ such that

$$
x_{n}=y_{n}+z_{n}+d_{n}
$$

and such that

$$
\mu\left(y_{n}\right)=\mu\left(y_{1}\right) \quad \text { for all } n \in \mathbb{N},
$$

with $\left\{z_{n}\right\}_{n=1}^{\infty}$ two-sided disjointly supported and $z_{n} \rightarrow 0$ in measure. By assumption, $\left\{z_{n}\right\}_{n=1}^{\infty}$ is a Banach-Saks sequence. Using Proposition 2.3, Lemma 2.9 and passing to a further subsequence and relabelling if necessary, we may assume that

$$
\frac{1}{n} \sum_{k=1}^{n} w_{k} \rightarrow 0
$$

in measure for every subsequence $\left\{w_{n}\right\}_{n=1}^{\infty} \subseteq\left\{y_{n}\right\}_{n=1}^{\infty}$. Suppose then that $\left\{w_{n}\right\}_{n=1}^{\infty} \subseteq\left\{y_{n}\right\}_{n=1}^{\infty}$ is an arbitrary subsequence. Set

$$
a_{n}:=\frac{1}{n} \sum_{k=1}^{n} w_{k}, \quad n \in \mathbb{N} .
$$

Since

$$
\mu\left(w_{n}\right)=\mu\left(y_{1}\right), \quad n \in \mathbb{N},
$$

it follows that

$$
a_{n} \preccurlyeq \mu(y), \quad n \in \mathbb{N} .
$$

Since $a_{n} \rightarrow 0$ in measure, Proposition 2.2 now shows that $\left\|a_{n}\right\|_{E} \rightarrow 0$. This implies that $\left\{y_{n}\right\}_{n=1}^{\infty}$ is a Banach-Saks sequence and, since $\left\{z_{n}\right\}_{n=1}^{\infty}$ is a Banach-Saks sequence, this suffices to establish the implication (ii) $\Rightarrow$ (i) in the case that $E^{\times} \subseteq L_{0}[0, \infty)$.

We now assume that $\tau(\mathbf{1})=\infty$ and that $E^{\times} \nsubseteq L_{0}[0, \infty)$. This implies that $L_{\infty}[0, \infty) \subseteq E^{\times}$, or equivalently, that $E \subseteq L_{1}[0, \infty)$. Using Proposition 2.12, and passing to subsequences and relabelling if necessary, we may assume that there are weakly null sequences $\left\{v_{n}\right\}_{n=1}^{\infty},\left\{w_{n}\right\}_{n=1}^{\infty}$ such 
that $x_{n}=v_{n}+w_{n}$ for all $n \in \mathbb{N}$, with $v_{n} \rightarrow 0$ in measure and $\left\{w_{n}\right\}_{n=1}^{\infty}$ $E$-equiintegrable at 0 . Passing to further subsequences if necessary, it follows from Lemma 2.4 and the assumption that $E(\mathcal{M}, \tau)$ has the $d$-Banach-Saks property that we may assume that $\left\{v_{n}\right\}_{n=1}^{\infty}$ is a Banach-Saks sequence.

It remains to show that $\left\{w_{n}\right\}_{n=1}^{\infty}$ contains a Banach-Saks subsequence. We set

$$
a_{n}:=\frac{1}{n} \sum_{k=1}^{n} w_{k}, \quad n \in \mathbb{N} .
$$

By Theorem 2.8, we may assume that there exists $y \in E(\mathcal{M}, \tau)$ such that $a_{n} \rightarrow y$ in measure, and since $\left\{w_{n}\right\}_{n=1}^{\infty}$ is weakly null, it follows that also $a_{n} \rightarrow 0$ weakly. From Lemma 2.9, it follows that $y=0$. We suppose that

$$
\left\|a_{n}\right\|_{E(\mathcal{M}, \tau)}>\varepsilon
$$

for infinitely many $n \in \mathbb{N}$. By Lemma 2.4, it may be assumed that the sequence $\left\{a_{n}\right\}_{n=1}^{\infty}$ is both left and right disjointly supported. By [CDS, Lemma 2.9], there exists a disjointly supported weakly null sequence $\left\{f_{n}\right\}_{n=1}^{\infty}$ $\subseteq E$ such that $\mu\left(f_{n}\right)=\mu\left(a_{n}\right)$ for all $n \in \mathbb{N}$. Further, since $a_{n} \rightarrow 0$ in measure, it follows that also $f_{n} \rightarrow 0$ in measure. Without loss of generality, we may assume that

$$
\left\|f_{n}\right\|_{E}>\varepsilon
$$

for all $n \in \mathbb{N}$. Since the sequence $\left\{w_{n}\right\}_{n=1}^{\infty}$ is $E$-equiintegrable at 0 , there exists $\delta>0$ such that

$$
\sup _{n \in \mathbb{N}}\left\|\mu\left(w_{n}\right) \chi_{[0, \delta)}\right\|_{E}<\varepsilon / 2 .
$$

Since

$$
\mu\left(f_{n}\right) \chi_{[0, \delta)}=\mu\left(a_{n}\right) \chi_{[0, \delta)} \nVdash\left(\frac{1}{n} \sum_{k=1}^{n} \mu\left(w_{k}\right)\right) \chi_{[0, \delta)}
$$

for all $n \in \mathbb{N}$, it also follows that

$$
\sup _{n \in \mathbb{N}}\left\|\mu\left(f_{n}\right) \chi_{[0, \delta)}\right\|_{E}<\varepsilon / 2 .
$$

Since $f_{n} \rightarrow 0$ in measure, we may assume without loss of generality that

$$
\sup _{n \in \mathbb{N}} \mu_{\delta}\left(f_{n}\right) \leq 1 .
$$

Set

$$
b_{n}:=f_{n} \chi_{(1, \infty)}\left(\left|f_{n}\right|\right), \quad c_{n}:=f_{n} \chi_{[0,1]}\left(\left|f_{n}\right|\right), \quad n \in \mathbb{N} .
$$

It follows from (2.10) and (2.9) that

$$
\left\|b_{n}\right\|_{E}<\varepsilon / 2
$$

for all $n \in \mathbb{N}$. Since the sequence $\left\{f_{n}\right\}_{n=1}^{\infty}$ is weakly null, it follows from [Fr, $82 \mathrm{G}]$ that the sequence $\left\{c_{n}\right\}_{n=1}^{\infty}$ is relatively weakly compact in $E$. Since 
$c_{n} \rightarrow 0$ in measure, Lemma 2.9 shows that $\left\{c_{n}\right\}_{n=1}^{\infty}$ is weakly null in $E$. Consequently, $\left\{c_{n}\right\}_{n=1}^{\infty}$ is weakly null in $L_{1}[0, \infty)$ by the assumption that $E \subseteq L_{1}[0, \infty)$. It follows from the well known Vitali convergence theorem that $\left\|c_{n}\right\|_{L_{1}[0, \infty)} \rightarrow 0$. Since $\mu\left(c_{n}\right) \leq 1$ for all $n \in \mathbb{N}$ and since $\mu\left(c_{n}\right) \rightarrow 0$ in measure, the separability of $E$ implies that $\left\|\mu\left(c_{n}\right) \chi_{[0,1)}\right\|_{E} \rightarrow 0$. Since $\mu\left(c_{n}\right) \rightarrow 0$ in measure, it follows that

$$
\left\|\mu\left(c_{n}\right) \chi_{[1, \infty)}\right\|_{L_{\infty}}=\mu_{1}\left(c_{n}\right) \rightarrow 0 .
$$

Since $\left\|\mu\left(c_{n}\right) \chi_{[1, \infty)}\right\|_{L_{1}} \rightarrow 0$, we deduce that

$$
\left\|\mu\left(c_{n}\right) \chi_{[1, \infty)}\right\|_{L_{1} \cap L_{\infty}} \rightarrow 0
$$

and consequently

$$
\left\|\mu\left(c_{n}\right) \chi_{[1, \infty)}\right\|_{E} \rightarrow 0
$$

since $L_{1} \cap L_{\infty}$ embeds continuously into $E$. It now follows that $\left\|c_{n}\right\|_{E} \rightarrow 0$ and, further, that

$$
\left\|f_{n}\right\|_{E}<\varepsilon
$$

for all sufficiently large $n \in \mathbb{N}$, and this contradiction completes the proof of the theorem.

We remark that, in the previous theorem, the assumption that $E$ should have the Fatou property cannot be omitted, even in the case that $\mathcal{M}$ is commutative, and $\tau(\mathbf{1})<\infty$. Indeed, it is shown in [DSS] that there exist Marcinkiewicz spaces on the interval $[0,1)$ whose separable part fails to have the Banach-Saks property. See the remarks following [DSS, Corollary 5.6]. However, it is well known that each bounded, disjointly supported sequence in the separable part of any Marcinkiewicz space on $[0,1)$ contains a subsequence equivalent to the standard unit vector basis of $c_{0}$. See, for example, [To, Proposition 1]. Consequently, the separable part of any Marcinkiewicz space on $[0,1)$ satisfies the second assertion of the previous theorem, but not the first.

We mention further that the commutative specialisation of Theorem 2.13 is stated in [DSS, Theorem 4.5]. However, the proof given there contains a gap, and is valid only under the additional assumption that $E^{\times} \subseteq L_{0}[0, \infty)$, as is also the case with [DSS, Proposition 4.2]. This gap is removed by the commutative specialisation of the present proof of Theorem 2.13.

THEOREM 2.14. If $E$ has the Fatou property, then the following conditions are equivalent:

(i) E has the Banach-Saks property;

(ii) $E(\mathcal{M}, \tau)$ has the Banach-Saks property for every semifinite von Neumann algebra $(\mathcal{M}, \tau)$. 
Proof. It clearly suffices to prove the implication (i) $\Rightarrow($ ii). We assume first that $(\mathcal{M}, \tau)$ is non-atomic, and suppose that $\left\{z_{n}\right\}_{n=1}^{\infty} \subseteq E(\mathcal{M}, \tau)$ is a weakly null two-sided disjointly supported sequence. By [CDS, Lemma 2.6], there exists a mutually disjoint, weakly null sequence $\left\{f_{n}\right\}_{n=1}^{\infty} \subseteq E$ such that $\mu\left(f_{n}\right)=\mu\left(z_{n}\right), n \geq 1$. It follows readily that

$$
\mu\left(\sum_{k=1}^{n} f_{n(k)}\right)=\mu\left(\sum_{k=1}^{n} z_{n(k)}\right)
$$

and this implies that

$$
\left\|\sum_{k=1}^{n} f_{n(k)}\right\|_{E}=\left\|\sum_{k=1}^{n} z_{n(k)}\right\|_{E(\mathcal{M}, \tau)}
$$

for any increasing sequence $n(k), k \in \mathbb{N}$. By assumption, the space $E$ has the Banach-Saks property and so $\left\{f_{n}\right\}$ is a Banach-Saks sequence. It follows immediately that $\left\{z_{n}\right\}$ is a Banach-Saks sequence. Now Theorem 2.13 implies that $E(\mathcal{M}, \tau)$ has the Banach-Saks property.

In the general case, set

$$
\mathcal{N}:=\mathcal{M} \otimes L_{\infty}(0,1), \quad \tau_{\mathcal{N}}:=\tau \otimes m,
$$

and identify $\mathcal{M}$ with the proper subalgebra $\mathcal{M} \otimes \chi_{[0,1]}$ via the mapping $\phi: x \mapsto x \otimes \chi_{[0,1]}, x \in \widetilde{\mathcal{M}}$. If $z \in E^{\times}\left(\mathcal{N}, \tau_{\mathcal{N}}\right)$, then there exists $y \in E^{\times}(\mathcal{M}, \tau)$ such that

$$
\mathcal{E}_{\mathcal{M} \otimes \chi_{[0,1)}} z=y \otimes \chi_{[0,1)}
$$

Consequently, using Lemma 1.2, for all $x \in E(\mathcal{M}, \tau)$ we get

$$
(\tau \otimes m)\left(\left(x \otimes \chi_{[0,1)}\right) z\right)=(\tau \otimes m)\left(\left(x \otimes \chi_{[0,1)}\right) \mathcal{E}_{\mathcal{M} \otimes \chi_{[0,1)}} z\right)=\tau(x y) .
$$

Noting that separability of $E$ implies that $E^{\times}\left(\mathcal{N}, \tau_{\mathcal{N}}\right)=E\left(\mathcal{N}, \tau_{\mathcal{N}}\right)^{*}$, and that $E^{\times}(\mathcal{M}, \tau)=E(\mathcal{M}, \tau)^{*}$, it follows that the restriction of $\phi$ to $E(\mathcal{M}, \tau)$ is continuous for the weak topologies on $E(\mathcal{M}, \tau), E\left(\mathcal{N}, \tau_{\mathcal{N}}\right)$ respectively. This implies that the restriction of $\phi$ to $E(\mathcal{M}, \tau)$ preserves weakly null sequences. Since $\left(\mathcal{N}, \tau_{\mathcal{N}}\right)$ is non-atomic, and since

$$
\|x\|_{E(\mathcal{M}, \tau)}=\left\|x \otimes \chi_{[0,1)}\right\|_{E\left(\mathcal{N}, \tau_{\mathcal{N}}\right)}, \quad x \in E(\mathcal{M}, \tau),
$$

the assertion of the theorem now follows from the first part of the proof.

It is classical [BS], [Ba], [Sz] that if $E=L_{p}, 1 \leq p<\infty$, then $E$ has the Banach-Saks property. Further, if $E$ is a separable Orlicz or Lorentz space, then also $E$ has the Banach-Saks property [DSS] (see also Theorem 3.14 below). Consequently, in each case, it follows from Theorem 2.14 that the space $E(\mathcal{M}, \tau)$ has the Banach-Saks property for all semifinite $(\mathcal{M}, \tau)$.

We remark that if $\mathcal{M}=\mathcal{B}(\mathcal{H})$ is the algebra of all bounded operators in some separable Hilbert space equipped with the standard trace, then the space $E(\mathcal{M}, \tau)$ is simply the Schatten ideal (or unitary matrix space) $\mathcal{C}_{E}$. 
Here we identify the underlying symmetric sequence space with the linear span in $E[0, \infty)$ of the vectors $e_{n}=\chi_{[n-1, n)}, n \in \mathbb{N}$. In this particular case, Theorem 2.14 reduces to [Ar, Corollary 3.6]. In the case of trace ideals, the approach of Arazy yields the stronger result that an arbitrary separable symmetric sequence space $E$ has the Banach-Saks property if and only if the Schatten ideal $\mathcal{C}_{E}$ has the Banach-Saks property. Suppose now that $\Phi$ is an Orlicz function. It has been shown by Rakov [Rak] that the separable part $l_{\Phi}^{0}$ of a non-separable Orlicz sequence space $l_{\Phi}^{0}$ always has the Banach-Saks property. It then follows from Arazy's result that the Schatten ideal $\mathcal{C}_{l_{\Phi}^{0}}$ also has the Banach-Saks property. However, it follows from [DSS, Theorem 5.5] that if $L_{\Phi}$ is a non-separable Orlicz space on any interval $[0, \alpha), 0<\alpha \leq \infty$, then the separable part $L_{\Phi}^{0}$ does not have the Banach-Saks property. Consequently, we obtain the following remark.

Proposition 2.15. If $L_{\Phi}$ is a non-separable Orlicz space, and if $(\mathcal{M}, \tau)$ is non-atomic, then $L_{\Phi}^{0}(\mathcal{M}, \tau)$ does not embed isomorphically in the trace ideal $\mathcal{C}_{l_{\Phi}^{0}}$.

3. $p$-Banach-Saks properties. We recall the following terminology [HRS].

Definition 3.1. Let $X$ be a Banach space and $1<p<\infty$.

(a) A weakly null sequence $\left\{x_{n}\right\}_{n=1}^{\infty} \subseteq X$ is said to be

(i) a $p$-Banach-Saks sequence if there exists a positive constant $C<\infty$ such that

$$
\limsup _{n \rightarrow \infty} n^{-1 / p}\left\|\sum_{j=1}^{n} y_{j}\right\| \leq C
$$

for all subsequences $\left\{y_{n}\right\}_{n=1}^{\infty} \subseteq\left\{x_{n}\right\}_{n=1}^{\infty}$;

(ii) a strong $p$-Banach-Saks sequence if

$$
\lim _{n \rightarrow \infty} n^{-1 / p}\left\|\sum_{j=1}^{n} y_{j}\right\|=0
$$

for all subsequences $\left\{y_{n}\right\}_{n=1}^{\infty} \subseteq\left\{x_{n}\right\}_{n=1}^{\infty}$.

(b) $X$ is said to have the $p$-Banach-Saks property (respectively, the strong $p$-Banach-Saks property) if each weakly null sequence in $X$ has a $p$-Banach-Saks subsequence (respectively, a strong $p$-BanachSaks subsequence).

We denote by $\left\{r_{n}\right\}_{n=1}^{\infty}$ the usual Rademacher sequence on $[0,1)$ defined by setting

$$
r_{n}(t)=\operatorname{sgn} \sin \left(2^{n} \pi t\right), \quad t \in[0,1), n=0,1, \ldots
$$


We recall [LT] that a Banach space $X$ is said to have type $p$ for some $1<p \leq 2$ if there exists a finite constant $M$ such that for every finite sequence $\left\{x_{j}\right\}_{j=1}^{n} \subseteq X$,

$$
\left\|\sum_{j=1}^{n} x_{j} r_{j}\right\|_{L_{2}([0,1), X)}=\left(\int_{0}^{1}\left\|\sum_{j=1}^{n} x_{j} r_{j}(t)\right\|_{X}^{2} d t\right)^{1 / 2} \leq M\left(\sum_{j=1}\left\|x_{j}\right\|_{X}^{p}\right)^{1 / p} .
$$

Let $1 \leq p, q \leq \infty$. A Banach lattice $X$ is said to be $p$-convex, respectively $q$-concave, if there exists a constant $M>0$ such that for every finite sequence $\left\{x_{j}\right\}_{j=1}^{n} \subseteq X$,

$$
\left\|\left(\sum_{j=1}^{n}\left|x_{j}\right|^{p}\right)^{1 / p}\right\|_{X} \leq M\left(\sum_{j=1}^{n}\left\|x_{j}\right\|_{X}^{p}\right)^{1 / p}
$$

respectively

$$
\left(\sum_{j=1}^{n}\left\|x_{j}\right\|_{X}^{q}\right)^{1 / q} \leq M\left\|\left(\sum_{j=1}^{n}\left|x_{j}\right|^{q}\right)^{1 / q}\right\|_{X} .
$$

The smallest such constant $M$ is called the modulus of $p$-convexity, respectively, modulus of q-concavity, of $X$. We remark that if $1<p \leq 2 \leq q<\infty$, and if $E$ is a symmetric Banach function space on $[0, \infty)$ which is $p$-convex and $q$-concave, then the argument of [LT, Proposition 1.d.8] shows that there exists on $E$ an equivalent symmetric norm with moduli of $p$-convexity and $q$-concavity both equal to 1 . With this equivalent norm, [LT, Theorem 1.f.1] shows that $E$ is uniformly convex with modulus of convexity of power type $q$ and uniformly smooth with modulus of smoothness of power type $p$. It follows, in particular, that $E$ is reflexive.

We remark that it has been shown by Rakov [Rak] that if a Banach space is of type $p, 1<p \leq 2$, then it has the $p$-Banach-Saks property.

Proposition 3.2. If $E$ is $p$-convex and $q$-concave for some $1<p \leq 2 \leq$ $q<\infty$, then $E(\mathcal{M}, \tau)$ has the $p$-Banach-Saks property.

Proof. By passing to an equivalent rearrangement-invariant norm on $E$ if necessary, we may assume that $E$ has modulus of $p$-convexity and modulus of $q$-concavity both equal to 1 . It then follows from [Xu1] that $E(\mathcal{M}, \tau)$ has type $p$. The assertion of the proposition now follows from the cited result of Rakov.

We remark that it follows from Theorem 2.13 (see also Corollary 3.7 below) and the classical theorem of Szlenk $[\mathrm{Sz}]$ that $L_{1}(\mathcal{M}, \tau)$ has the BanachSaks property (in fact, as shown in [BD], any von Neumann algebra predual has the Banach-Saks property). We note that Proposition 3.2 implies that $L_{p}(\mathcal{M}, \tau)$ has the $p$-Banach-Saks property for $1<p \leq 2$. In the case that 
$(\mathcal{M}, \tau)$ is finite, this was shown in [HRS, Proposition 4.6] quite independently of Rakov's result. It was noted further in [HRS] (see also [Su]) that if $(\mathcal{M}, \tau)$ is finite, then $L_{p}(\mathcal{M}, \tau)$ has the 2-Banach-Saks property for $p>2$ and that this is best possible.

The definition which follows extends the classical notion of uniform equiintegrability to non-commutative spaces of measurable operators.

Definition 3.3. If $K \subseteq E(\mathcal{M}, \tau)$ is bounded, then $K$ is said to be E-equiintegrable if

$$
\sup _{x \in K}\left\{\left\|e_{n} x e_{n}\right\|_{E(\mathcal{M}, \tau)}\right\} \rightarrow 0
$$

for every system $\left\{e_{n}\right\}_{n=1}^{\infty} \subseteq \mathcal{M}$ of projections with $e_{n} \downarrow 0$.

The preceding definition was introduced by Randrianantoanina [Ran2] (see also [Ran1]), although related notions had been earlier considered in [CS]. If $E=L_{1}[0, \infty)$, then the well known criterion of Akemann [Ta] (see also $[\mathrm{RX}]$ ) asserts that the $E$-equiintegrable subsets of $L_{1}(\mathcal{M}, \tau)$ are precisely those which are relatively weakly compact. Equiintegrable sets in the Haagerup $L_{p}$-spaces have been studied in some detail in [RX] via ultraproduct techniques, but these methods do not apply in the present setting.

We observe that it follows immediately from Proposition 2.11 that any bounded $E$-equintegrable sequence is $E$-equintegrable at 0 in the sense of Definition 2.10 and that the two notions coincide if the trace $(\mathcal{M}, \tau)$ is finite. We note further that if the sequence $\left\{y_{k}\right\}_{k=1}^{\infty}$ is equimeasurable, and if $\tau(\mathbf{1})<\infty$, then $\left\{y_{k}\right\}_{k=1}^{\infty}$ is $E$-equiintegrable. In fact, the submajorisation (see $[\mathrm{CS}]$ )

$$
\text { ye } \mu \mu(y) \chi_{[0, \tau(e))}
$$

together with the equimeasurability of the sequence $\left\{y_{k}\right\}_{k=1}^{\infty}$, the finiteness of the trace $\tau$ and the separability of $E$ immediately yields

$$
\sup _{k}\left\|e_{n} y_{k} e_{n}\right\|_{E(\mathcal{M}, \tau)} \leq\left\|\mu(y) \chi_{\left[0, \tau\left(e_{n}\right)\right)}\right\|_{E} \rightarrow 0
$$

whenever $e_{n} \downarrow 0$. It is clear that this assertion fails in the case of $\tau(\mathbf{1})=\infty$.

Lemma 3.4. Let $z \in \widetilde{\mathcal{M}}$ and let $e, f \in \mathcal{M}$ be self-adjoint projections.

(i) $|z e|=|| z|e|=e|z e| e$.

(ii) If $e \leq f$, then $\mu(z e) \leq \mu(z f)$.

Proof. (i) It follows from the equality

$$
|z e|^{2}=e|z|^{2} e=|| z|e|^{2}
$$

that $|z e|=|| z|e|$ for all $z \in \widetilde{\mathcal{M}}$. If $p$ denotes the right support of $z e$ (which is the right support of $|z e|$ ), then it is clear that $p \leq e$ and this implies $e|z e| e=|z e|$. 
(ii) If $e \leq f \in \mathcal{M}$ are self-adjoint projections with $e \leq f$, then

$$
\begin{aligned}
\mu(z e) & =\mu(|z| e)=\mu(e|z|)=\mu^{1 / 2}(|z| e|z|) \\
& \leq \mu^{1 / 2}(|z| f|z|)=\mu(f|z|)=\mu(|z| f)=\mu(z f) .
\end{aligned}
$$

LEMMA 3.5. Suppose that $\mathcal{M}$ is non-atomic and that $\left\{x_{n}\right\}_{n=1}^{\infty} \subseteq E(\mathcal{M}, \tau)$ is E-equiintegrable. If

$$
x_{n}=y_{n}+z_{n}, \quad n \in \mathbb{N},
$$

where the sequence $\left\{y_{n}\right\}_{n=1}^{\infty}$ is equimeasurable and $\left\{z_{n}\right\}_{n=1}^{\infty}$ converges to 0 for the measure topology, then $\left\|z_{n}\right\|_{E(\mathcal{M}, \tau)} \rightarrow 0$.

Proof. We show first that

$$
\left\|e_{n} z_{n} e_{n}\right\|_{E(\mathcal{M}, \tau)}=\left\|e_{n} z_{n}^{*} e_{n}\right\|_{E(\mathcal{M}, \tau)} \rightarrow 0
$$

for any sequence $\left\{e_{n}\right\}_{n=1}^{\infty}$ of projections for which $\sum_{n=1}^{\infty} \tau\left(e_{n}\right)<\infty$. In fact,

$$
\begin{aligned}
\left\|e_{n} z_{n} e_{n}\right\|_{E(\mathcal{M}, \tau) \leq} & \left\|\left(\vee_{k \geq n} e_{k}\right) z_{n}\left(\vee_{k \geq n} e_{k}\right)\right\|_{E(\mathcal{M}, \tau)} \\
\leq & \left\|\left(\vee_{k \geq n} e_{k}\right) x_{n}\left(\vee_{k \geq n} e_{k}\right)\right\|_{E(\mathcal{M}, \tau)}+\| y_{n}\left(\left(\vee_{k \geq n} e_{k}\right) \|_{E(\mathcal{M}, \tau)}\right. \\
\leq & \sup _{j}\left\|\left(\vee_{k \geq n} e_{k}\right) x_{j}\left(\vee_{k \geq n} e_{k}\right)\right\|_{E(\mathcal{M}, \tau)} \\
& +\left\|\mu\left(y_{1}\right) \chi_{\left[0, \sum_{k \geq n} \tau\left(e_{k}\right)\right)}\right\|_{E},
\end{aligned}
$$

where we have again used the submajorisation

$$
\mu(y e) \preccurlyeq \mu(y) \mu(e) .
$$

Since $\vee_{k \geq n} e_{k} \downarrow 0$, the assertion follows from the separability of $E$ and the assumption that the sequence $\left\{x_{n}\right\}_{n=1}^{\infty}$ is $E$-equiintegrable.

We show next that

$$
\left\|z_{n} f_{n}\right\|_{E(\mathcal{M}, \tau)} \rightarrow 0, \quad\left\|z_{n}^{*} f_{n}\right\|_{E(\mathcal{M}, \tau)} \rightarrow 0
$$

for every sequence $\left\{f_{n}\right\}_{n=1}^{\infty} \subseteq \mathcal{M}$ of projections with $\sup _{n} \tau\left(f_{n}\right)<\infty$. Let us first remark that, if $z \in E(\mathcal{M}, \tau)$, if $\varepsilon, \delta>0$ and if $p, q$ are projections with $\tau(\mathbf{1}-p), \tau(\mathbf{1}-q)<\delta$ and are such that

$$
\|z p\|_{E(\mathcal{M}, \tau)}<\varepsilon, \quad\left\|z^{*} q\right\|_{E(\mathcal{M}, \tau)}<\varepsilon
$$

then, setting $e:=\mathbf{1}-(\mathbf{1}-p) \vee(\mathbf{1}-q)$, it is easily checked that $\tau(\mathbf{1}-e)<2 \delta$ and

$$
\|z e\|_{E(\mathcal{M}, \tau)}<\varepsilon, \quad\left\|z^{*} e\right\|_{E(\mathcal{M}, \tau)}<\varepsilon .
$$

Since $z_{n}, z_{n}^{*} \rightarrow 0$ in measure, using the preceding remark and passing to a subsequence and relabelling if necessary, we may assume that there exists a sequence $\left\{e_{n}\right\}_{n=1}^{\infty}$ of projections such that $\sum_{n=1}^{\infty} \tau\left(\mathbf{1}-e_{n}\right)<\infty$ and such that

$$
\left\|z_{n} e_{n}\right\|_{\mathcal{M}} \rightarrow 0, \quad\left\|z_{n}^{*} e_{n}\right\|_{\mathcal{M}} \rightarrow 0
$$


By (3.1), we may assume that

$$
\left\|\left(\mathbf{1}-e_{n}\right) z_{n}\left(\mathbf{1}-e_{n}\right)\right\|_{E(\mathcal{M}, \tau)} \rightarrow 0, \quad\left\|\left(\mathbf{1}-e_{n}\right) z_{n}^{*}\left(\mathbf{1}-e_{n}\right)\right\|_{E(\mathcal{M}, \tau)} \rightarrow 0 .
$$

If $\left\{f_{n}\right\}_{n=1}^{\infty}$ is any sequence of projections with $\sup _{n} \tau\left(f_{n}\right)<\infty$ then

$$
\begin{aligned}
\left\|z_{n} f_{n}\right\|_{E(\mathcal{M}, \tau) \leq} & \left\|z_{n} e_{n} f_{n}\right\|_{E(\mathcal{M}, \tau)}+\left\|e_{n} z_{n}\left(\mathbf{1}-e_{n}\right) f_{n}\right\|_{E(\mathcal{M}, \tau)} \\
& +\left\|\left(\mathbf{1}-e_{n}\right) z_{n}\left(\mathbf{1}-e_{n}\right) f_{n}\right\|_{E(\mathcal{M}, \tau)} \\
\leq & \left\|z_{n} e_{n} f_{n}\right\|_{L_{1}(\mathcal{M}, \tau) \cap \mathcal{M}}+\left\|f_{n}\left(\mathbf{1}-e_{n}\right) z_{n}^{*} e_{n}\right\|_{L_{1}(\mathcal{M}, \tau) \cap \mathcal{M}} \\
& +\left\|\left(\mathbf{1}-e_{n}\right) z_{n}\left(\mathbf{1}-e_{n}\right)\right\|_{E(\mathcal{M}, \tau)} \\
\leq & \max \left\{1, \sup _{n} \tau\left(f_{n}\right)\right\}\left(\left\|z_{n} e_{n}\right\|_{\mathcal{M}}+\left\|z_{n}^{*} e_{n}\right\|_{\mathcal{M})}\right. \\
& +\left\|\left(\mathbf{1}-e_{n}\right) z_{n}\left(\mathbf{1}-e_{n}\right)\right\|_{E(\mathcal{M}, \tau)} \rightarrow 0 .
\end{aligned}
$$

The preceding argument applies equally with $z_{n}$ replaced by $z_{n}^{*}$, and shows that each subsequence of each of the sequences $\left\{z_{n} f_{n}\right\}_{n=1}^{\infty},\left\{z_{n}^{*} f_{n}\right\}_{n=1}^{\infty}$ contains a null subsequence, and this suffices to establish (3.2).

Suppose now that there exists $\varepsilon>0$ such that

$$
\left\|z_{n}\right\|_{E(\mathcal{M}, \tau)}>2 \varepsilon, \quad n \in \mathbb{N} .
$$

Since the sequence $\left\{x_{n}\right\}_{n=1}^{\infty}$ is $E$-equiintegrable, there exists a projection $p$ with $\tau(p)<\infty$ and such that

$$
\sup _{n}\left\|(\mathbf{1}-p) x_{n}(\mathbf{1}-p)\right\|_{E(\mathcal{M}, \tau)}<\varepsilon / 8
$$

To see that this is the case, suppose that $\left\{e_{\alpha}\right\}_{\alpha \in \mathcal{A}}$ is a mutually disjoint family of projections such that $\tau\left(e_{\alpha}\right)<\infty$ for all $\alpha \in \mathcal{A}$ and $\sum_{\alpha \in \mathcal{A}} e_{\alpha}=\mathbf{1}$. By a similar argument to those in [Xu2, Lemma 5.7 and proof of Theorem 5.1] (see also [Ran2]), there exists an at most countable subset $\left\{e_{n}\right\}_{n=1}^{\infty}$ such that

$$
x_{n} e_{\alpha}=e_{\alpha} x_{n}=y_{n} e_{\alpha}=e_{\alpha} y_{n}=z_{n} e_{\alpha}=e_{\alpha} z_{n}=0
$$

whenever $e_{\alpha} \notin\left\{e_{n}\right\}_{n=1}^{\infty}$. Set $e:=\sum_{n=1}^{\infty} e_{n}$. Replacing $\mathcal{M}$ by $e \mathcal{M} e$ and $\tau$ by its restriction to $e \mathcal{M} e$, we may suppose that $e=1$. If we now set $p_{n}:=\sum_{k \geq n} e_{k}$, then it is clear that $p_{n} \downarrow 0$ and $\tau\left(\mathbf{1}-p_{n}\right)<\infty$ for all $n \in \mathbb{N}$. Using the E-equintegrability of $\left\{x_{n}\right\}_{n=1}^{\infty}$, this clearly suffices to establish (3.4). From (3.2), with $f_{n}$ taken to be $\mathbf{1}-p$ for all $n \geq 1$, and using (3.3), we may assume that

$$
\left\|(\mathbf{1}-p) z_{n}(\mathbf{1}-p)\right\|_{E(\mathcal{M}, \tau)} \geq \varepsilon
$$

for all sufficiently large $n \in \mathbb{N}$. For all such $n \in \mathbb{N}$, it then follows from (3.4) that

$$
\left\|(\mathbf{1}-p) y_{n}(\mathbf{1}-p)\right\|_{E(\mathcal{M}, \tau)} \geq 7 \varepsilon / 8 .
$$

Since $E$ is separable, there exist positive real numbers $r \leq s$ such that

$$
\left\|\mu\left(y_{1}\right) \chi_{[0, r)}\right\|_{E}<\varepsilon / 16, \quad\left\|\mu\left(y_{1}\right) \chi_{[s, \infty)}\right\|_{E}<\varepsilon / 16 .
$$


Using the fact that $(\mathcal{M}, \tau)$ is non-atomic, and the remark following Proposition 1.1, for each $n \in \mathbb{N}$, there exist disjoint projections $e_{n}^{(1)}, e_{n}^{(2)}, e_{n}^{(3)}$ in $\mathcal{M}$ such that

$$
\begin{gathered}
\tau\left(e_{n}^{(1)}\right)=r, \quad \tau\left(e_{n}^{(2)}\right)=s-r \\
(\mathbf{1}-p) y_{n}(\mathbf{1}-p)=(\mathbf{1}-p) y_{n}(\mathbf{1}-p) e_{n}^{(1)}+(\mathbf{1}-p) y_{n}(\mathbf{1}-p) e_{n}^{(2)} \\
+(\mathbf{1}-p) y_{n}(\mathbf{1}-p) e_{n}^{(3)}
\end{gathered}
$$

and

$$
\begin{aligned}
\mu\left((\mathbf{1}-p) y_{n}(\mathbf{1}-p) e_{n}^{(1)}\right) & =\mu\left(\mu\left((\mathbf{1}-p) y_{n}(\mathbf{1}-p)\right) \chi_{[0, r)}\right) \\
& \leq \mu\left(\mu\left(y_{1}\right) \chi_{[0, r)}\right), \\
\mu\left((\mathbf{1}-p) y_{n}(\mathbf{1}-p) e_{n}^{(2)}\right) & =\mu\left(\mu\left((\mathbf{1}-p) y_{n}(\mathbf{1}-p)\right) \chi_{[r, s)}\right), \\
\mu\left((\mathbf{1}-p) y_{n}(\mathbf{1}-p) e_{n}^{(3)}\right) & =\mu\left(\mu\left((\mathbf{1}-p) y_{n}(\mathbf{1}-p)\right) \chi_{[s, \infty)}\right) \\
& \leq \mu\left(\mu\left(y_{1}\right) \chi_{[s, \infty)}\right) .
\end{aligned}
$$

From (3.7) and (3.8), it follows immediately that

$$
\begin{aligned}
\left\|(\mathbf{1}-p) y_{n}(\mathbf{1}-p) e_{n}^{(1)}\right\|_{E(\mathcal{M}, \tau)} & <\varepsilon / 16, \\
\left\|(\mathbf{1}-p) y_{n}(\mathbf{1}-p) e_{n}^{(3)}\right\|_{E(\mathcal{M}, \tau)} & <\varepsilon / 16 .
\end{aligned}
$$

From (3.6) and (3.9), we obtain

$$
\left\|(\mathbf{1}-p) y_{n}(\mathbf{1}-p) e_{n}^{(2)}\right\|_{E(\mathcal{M}, \tau)} \geq 6 \varepsilon / 8
$$

for all sufficiently large $n$. Since $\tau(p)<\infty$ and since $\tau\left(e_{n}^{(2)}\right)=s-r$ for all $n \in \mathbb{N}$, it follows from (3.2) that $\left\|z_{n} p\right\|_{E(\mathcal{M}, \tau)} \rightarrow 0$ and $\left\|z_{n} e_{n}^{(2)}\right\|_{E(\mathcal{M}, \tau)} \rightarrow 0$. This in turn implies that

$$
\left\|(\mathbf{1}-p) z_{n}(\mathbf{1}-p) e_{n}^{(2)}\right\|_{E(\mathcal{M}, \tau)} \leq\left\|z_{n} e_{n}^{(2)}\right\|_{E(\mathcal{M}, \tau)}+\left\|z_{n} p\right\|_{E(\mathcal{M}, \tau)} \rightarrow 0 .
$$

It now follows from (3.10) and (3.11) that

$$
\begin{aligned}
\underset{n}{\limsup \|(\mathbf{1}-p)\left(y_{n}+\right.} & \left.z_{n}\right)(\mathbf{1}-p) \|_{E(\mathcal{M}, \tau)} \\
& \geq \underset{n}{\limsup }\left\|(\mathbf{1}-p)\left(y_{n}+z_{n}\right)(\mathbf{1}-p) e_{n}^{(2)}\right\|_{E(\mathcal{M}, \tau)} \\
& \geq \underset{n}{\lim \sup }\left\|(\mathbf{1}-p) y_{n}(\mathbf{1}-p) e_{n}^{(2)}\right\|_{E(\mathcal{M}, \tau)} \geq 6 \varepsilon / 8 .
\end{aligned}
$$

This contradicts (3.4) and serves to complete the proof of the lemma.

By taking $y_{n}=0$ for all $n \in \mathbb{N}$ in the preceding Lemma 3.5, we obtain the following consequence, which in the commutative setting reduces to a well known theorem of Vitali. An alternative proof of this corollary may be based directly on Lemma 2.4 . 
Corollary 3.6. If $\left\{x_{n}\right\}_{n=1}^{\infty} \subseteq E(\mathcal{M}, \tau)$ is bounded and E-equiintegrable, then $\left\|x_{n}\right\|_{E(\mathcal{M}, \tau)} \rightarrow 0$ if and only if $x_{n} \rightarrow 0$ for the measure topology.

COROLlary 3.7. Suppose that $E$ has the Fatou property and that $(\mathcal{M}, \tau)$ is non-atomic. If $\left\{x_{n}\right\}_{n=1}^{\infty} \subseteq E(\mathcal{M}, \tau)$ is weakly null and E-equiintegrable, then $\left\{x_{n}\right\}_{n=1}^{\infty}$ contains a Banach-Saks subsequence.

Proof. It follows from Proposition 2.7 and Lemma 3.5 that we may, in addition, assume that the sequence $\left\{x_{n}\right\}_{n=1}^{\infty}$ is equimeasurable. Passing to a subsequence and relabelling, we may assume by an appeal to Theorem 2.8 that there exists $y \in L_{1}(\mathcal{M}, \tau)+\mathcal{M}$ such that

$$
n^{-1} \sum_{j=1}^{n} x_{j} \rightarrow y
$$

in measure. Since $\left\{x_{n}\right\}_{n=1}^{\infty}$ is weakly null, so also is $\left\{n^{-1} \sum_{j=1}^{n} x_{j}\right\}_{n=1}^{\infty}$. From Lemma 2.9, it follows that $y=0$. We now observe that the sequence $\left\{n^{-1} \sum_{j=1}^{n} x_{j}\right\}_{n=1}^{\infty}$ is $E$-equiintegrable. Indeed, for any sequence $\left\{e_{n}\right\}_{n=1}^{\infty}$ of projections in $\mathcal{M}$ with $e_{n} \downarrow 0$,

$$
\sup _{k \in \mathbb{N}}\left\|e_{n}\left(k^{-1} \sum_{j=1}^{k} x_{j}\right) e_{n}\right\|_{E(\mathcal{M}, \tau)} \leq \sup _{j \in \mathbb{N}}\left\|e_{n} x_{j} e_{n}\right\|_{E(\mathcal{M}, \tau)} \rightarrow 0 .
$$

It now follows from Corollary 3.6 that

$$
n^{-1}\left\|\sum_{j=1}^{n} x_{j}\right\|_{E(\mathcal{M}, \tau)} \rightarrow 0,
$$

and this suffices to complete the proof.

If $E=L_{1}[0, \infty)$, then, via the Akemann criterion (see the remarks following Definition 3.3), the preceding Corollary 3.7 is a non-commutative extension of the classical theorem of Szlenk [Sz], and the method of the preceding corollary is quite different from the lifting argument based on Theorem 2.13. We remark, however, that the commutative specialisation of Corollary 3.7 is valid under only the assumption that $E$ is separable, without requiring that $E$ have the Fatou property. See [DSS, Theorem 4.10].

Before proceeding, we recall that a sequence $\left\{x_{n}\right\}_{n=1}^{\infty}$ in a Banach space $X$ is called unconditional if there exists a constant $K>0$ such that

$$
\left\|\sum_{i=1}^{n} \alpha_{i} c_{i} x_{i}\right\| \leq K\left\|\sum_{i=1}^{n} c_{i} x_{i}\right\|
$$

for all $n \in \mathbb{N}$ and for all scalars $c_{1}, \ldots, c_{n}$ and $\alpha_{1}, \ldots \alpha_{n}$ with $\left|\alpha_{i}\right|=1$ for all $1 \leq i \leq n$. Such a sequence will be called $K$-unconditional. A set $W$ in a Banach space $X$ is said to be seminormalised if there exist constants $0<C_{1} \leq C_{2}<\infty$ such that $C_{1} \leq\|x\|_{X} \leq C_{2}$ for all $x \in W$. We shall 
need the following consequence of a well known result due to Brunel and Sucheston. See [BrS1,2], [HRS].

LEMMA 3.8. Let $\left\{x_{j}\right\}_{j=1}^{\infty}$ be a seminormalised weakly null sequence in a Banach space $X$. There exists a subsequence $\left\{y_{j}\right\}_{j=1}^{\infty} \subseteq\left\{x_{j}\right\}_{j=1}^{\infty}$ such that, for every $k \geq 1$, the sequence $\left\{y_{j}\right\}_{j=k}^{2^{k}}$ is 4-unconditional.

Theorem 3.9. Suppose that $(\mathcal{M}, \tau)$ is non-atomic. If $E$ is p-convex and $q$-concave for some $1<p<2 \leq q<\infty$, then each weakly null, E-equiintegrable sequence in $E(\mathcal{M}, \tau)$ contains a strong p-Banach-Saks subsequence.

Proof. It is clear that we may assume in addition that $x_{n}^{*}=x_{n}$ for all $n \in \mathbb{N}$. Let us note first that it follows from [LT, Theorems 1.f.10 and 1.c.4] that $E$ is separable and has the Fatou property. Using Proposition 2.7 and appealing to Lemma 3.5, it is readily seen that we may, in addition, assume that the sequence $\left\{x_{n}\right\}_{n=1}^{\infty}$ is equimeasurable. It is clear that we may assume that $\left\|x_{n}\right\|_{E(\mathcal{M}, \tau)}=\left\|x_{1}\right\|_{E(\mathcal{M}, \tau)}>0$ for all $n \in \mathbb{N}$. Since $\mathcal{M}$ is nonatomic, by Proposition 1.1 there exist rearrangement-preserving mappings $J_{n}: E \rightarrow E(\mathcal{M}, \tau), n \in \mathbb{N}$, such that $J_{n}\left(\mu\left(x_{n}\right)\right)=\left|x_{n}\right|$; let $u_{n}$ be (selfadjoint) unitary operators such that $x_{n}=u_{n}\left|x_{n}\right|$ for all $n \in \mathbb{N}$. Given $\varepsilon>0$, and using the separability of $E$, there exist $0<\delta \leq \Delta<\infty$ such that

$$
\left\|\mu\left(x_{1}\right)\left(\chi_{[0, \delta)}+\chi_{[\Delta, \infty)}\right)\right\|_{E}<\varepsilon, \quad n \in \mathbb{N} .
$$

Setting $e_{n}=J_{n}\left(\chi_{[\delta, \Delta)}\right)$, observe that $x_{n} e_{n}=e_{n} x_{n}$ for all $n \in \mathbb{N}$, that

$$
\mu\left(x_{n} e_{n}\right)=\mu\left(\mu\left(x_{1}\right) \chi_{[\delta, \Delta)}\right), \quad n \in \mathbb{N},
$$

and that

$$
\mu\left(x_{n}\left(1-e_{n}\right)\right)=\mu\left(\mu\left(x_{1}\right)\right)\left(\chi_{[0, \delta)}+\chi_{(\Delta, \infty)}\right), \quad n \in \mathbb{N} .
$$

It follows immediately that

$$
\sup _{n}\left\|x_{n}\left(1-e_{n}\right)\right\|_{E(\mathcal{M}, \tau)}<\varepsilon .
$$

Since the sequence $\left\{x_{n}\right\}_{n=1}^{\infty}$ is $E$-equiintegrable, and using the same argument as in the proof of Lemma 3.5, we may assume that there exists a projection $e \in \mathcal{M}$ with $\tau(\mathbf{1}-e)<\infty$ such that

$$
\left\|e x_{n} e\right\|_{E(\mathcal{M}, \tau)}<\varepsilon, \quad n \in \mathbb{N} .
$$

By (3.12), it follows that

$$
\left\|e x_{n}\left(\mathbf{1}-e_{n}\right) e\right\|_{E(\mathcal{M}, \tau)} \leq\left\|x_{n}\left(\mathbf{1}-e_{n}\right)\right\|_{E(\mathcal{M}, \tau)}<\varepsilon, \quad n \in \mathbb{N},
$$

and consequently, by (3.13),

$$
\left\|e x_{n} e_{n} e\right\|_{E(\mathcal{M}, \tau)}<2 \varepsilon, \quad n \in \mathbb{N} .
$$


Further, observe that

$$
\sup _{n \in \mathbb{N}}\left\|x_{n} e_{n}(\mathbf{1}-e)\right\|_{\mathcal{M}} \leq \sup _{n \in \mathbb{N}}\left\|x_{n} e_{n}\right\|_{\mathcal{M}}=\mu_{\delta}\left(x_{1}\right) .
$$

Using Lemma 3.8, and passing to a subsequence if necessary and relabelling, we may assume further that the sequence $\left\{x_{n}\right\}_{n=k}^{2^{k}}$ is 4-unconditional for each $k \geq 1$. Given $n \geq 1$, let $k \in \mathbb{N}$ satisfy $2^{k-1} \leq n<2^{k}$. Since

$$
n^{-1 / p}\left\|\sum_{i=1}^{k-1} x_{i}\right\|_{E(\mathcal{M}, \tau)} \leq \frac{\ln n}{n^{1 / p} \ln 2}\left\|x_{1}\right\|_{E(\mathcal{M}, \tau)} \rightarrow 0,
$$

it will suffice to show that

$$
\lim _{n \rightarrow \infty} n^{-1 / p}\left\|\sum_{i=k}^{n} x_{i}\right\|_{E(\mathcal{M}, \tau)}=0
$$

whenever $n, k \in \mathbb{N}$ satisfy $k \leq n<2^{k}$. From the assumption that $\left\{x_{n}\right\}_{n=k}^{2^{k}}$ is 4-unconditional, it follows that

$$
\left\|\sum_{i=k}^{n} x_{i}\right\|_{E(\mathcal{M}, \tau)} \leq 4\left\|\sum_{i=k}^{n} x_{i} r_{i}\right\|_{L_{2}([0,1), E(\mathcal{M}, \tau))} .
$$

Setting $f=\mathbf{1}-e$, it is clear that

$$
\begin{aligned}
& \left\|\sum_{i=k}^{n} x_{i} r_{i}\right\|_{L_{2}([0,1), E(\mathcal{M}, \tau))} \\
& \leq\left\|\sum_{i=k}^{n} x_{i}\left(\mathbf{1}-e_{i}\right) r_{i}\right\|_{L_{2}([0,1), E(\mathcal{M}, \tau))}+\left\|\sum_{i=k}^{n} e x_{i} e_{i} e r_{i}\right\|_{L_{2}([0,1), E(\mathcal{M}, \tau))} \\
& \quad+\left\|\sum_{i=k}^{n} x_{i} e_{i} f r_{i}\right\|_{L_{2}([0,1), E(\mathcal{M}, \tau))}+\left\|\sum_{i=k}^{n} f x_{i} e_{i} e r_{i}\right\|_{L_{2}([0,1), E(\mathcal{M}, \tau))}
\end{aligned}
$$

We now estimate each of the summands on the right hand side. It follows from the given assumptions on $E$ and from $[\mathrm{Xu} 1]$ that $E(\mathcal{M}, \tau)$ is of type $p$. Using (3.12), (3.14) and letting $M_{p}$ denote the constant in the definition of type, we obtain

$$
\begin{aligned}
\left\|\sum_{i=k}^{n} x_{i}\left(\mathbf{1}-e_{i}\right) r_{i}\right\|_{L_{2}([0,1), E(\mathcal{M}, \tau))} & \leq M_{p}(n-k)^{1 / p} \varepsilon \\
\left\|\sum_{i=k}^{n} e x_{i} e_{i} e r_{i}\right\|_{L_{2}([0,1), E(\mathcal{M}, \tau))} & \leq 2 M_{p}(n-k)^{1 / p} \varepsilon .
\end{aligned}
$$

It follows from the fact that $\tau(f)<\infty$ and from [LT, Remark 2, p. 133] that

$$
L_{q}([0, \tau(f))) \subseteq E[0, \tau(f))
$$


with embedding constant $C_{1}=C_{1}(\tau(f))$ which depends on $\tau(f)$. For brevity, we denote by $\left(\mathcal{M}^{f}, \tau^{f}\right)$ the von Neumann algebra $f \mathcal{M} f$ equipped with the (finite) trace $\tau(f \cdot f)$. Since $x_{i} e_{i} \in \mathcal{M}, i \in \mathbb{N}$, Lemma 3.4(i) shows that, for all $\omega \in[0,1)$,

$$
\left|\sum_{i=k}^{n} x_{i} e_{i} r_{i}(\omega) f\right| \in \mathcal{M}^{f} \subseteq L_{q}\left(\mathcal{M}^{f}, \tau^{f}\right) \subseteq E\left(\mathcal{M}^{f}, \tau^{f}\right) .
$$

Since $q \geq 2$, it follows from [Fa] that $L_{q}\left(\mathcal{M}^{f}, \tau^{f}\right)$ has type 2 . Let $M_{2}$ denote the type 2 constant. Using (3.15), (3.20), we obtain

$$
\begin{aligned}
\left\|\sum_{i=k}^{n} x_{i} e_{i} f r_{i}\right\|_{L_{2}([0,1), E(\mathcal{M}, \tau))} & =\left\|\sum_{i=k}^{n} x_{i} e_{i} f r_{i}\right\|_{L_{2}\left([0,1), E\left(\mathcal{M}^{f}, \tau^{f}\right)\right)} \\
& \leq C_{1}\left\|\sum_{i=k}^{n} x_{i} e_{i} f r_{i}\right\|_{L_{2}\left([0,1), L_{q}\left(\mathcal{M}^{f}, \tau^{f}\right)\right)} \\
& \leq M_{2} C_{1}\left(\sum_{i=k}^{n}\left\|x_{i} e_{i} f\right\|_{L_{q}\left(\mathcal{M}^{f}, \tau^{f}\right)}^{2}\right)^{1 / 2} \\
& \leq M_{2} C_{1}\left(\sup _{k \leq i \leq n}\left\|x_{i} e_{i} f\right\|_{\left.\mathcal{M}^{f}\right)(n-k)^{1 / 2} \tau(f)^{1 / q}}\right. \\
& \leq M_{2} C_{1} \mu_{\delta}\left(x_{1}\right)(n-k)^{1 / 2} \tau(f)^{1 / q} .
\end{aligned}
$$

Passing to adjoints, we obtain similarly

$$
\left\|\sum_{i=k}^{n} f x_{i} e_{i} e r_{i}\right\|_{L_{2}([0,1), E(\mathcal{M}, \tau))} \leq M_{2} C_{1} K(n-k)^{1 / 2} \tau(f)^{1 / q} .
$$

If we combine (3.17), (3.18), (3.19), (3.21), (3.22), it follows that

$$
n^{-1 / p}\left\|\sum_{i=k}^{n} x_{i}\right\|_{E(\mathcal{M}, \tau)} \leq 12 M_{p} \varepsilon+8 C_{1} M_{2} \mu_{\delta}\left(x_{1}\right) \tau(f)^{1 / q} n^{1 / 2-1 / p}
$$

for all $n \in \mathbb{N}$. Since $p<2$, this yields

$$
\limsup _{n \rightarrow \infty} n^{-1 / p}\left\|\sum_{i=k}^{n} x_{i}\right\|_{E(\mathcal{M}, \tau)} \leq 12 M_{p} \varepsilon .
$$

As $\varepsilon>0$ is arbitrary, the assertion of the theorem follows.

LemmA 3.10. If $\left\{x_{n}\right\}_{n=1}^{\infty} \subseteq \widetilde{\mathcal{M}}$ converges to 0 in measure, and if $p>0$, then there exists a subsequence $\left\{y_{n}\right\}_{n=1}^{\infty} \subseteq\left\{x_{n}\right\}_{n=1}^{\infty}$ such that $n^{-1 / p} \sum_{j=1}^{n} w_{j}$ $\rightarrow 0$ in measure for all further subsequences $\left\{w_{n}\right\}_{n=1}^{\infty} \subseteq\left\{y_{n}\right\}_{n=1}^{\infty}$.

Proof. It will suffice to show that if $\varepsilon>0, \delta>0$ are given, and if $N(\varepsilon, \delta)$ is the corresponding neighbourhood of 0 for the measure topology, 
then there exists a subsequence $\left\{y_{n}\right\}_{n=1}^{\infty} \subseteq\left\{x_{n}\right\}_{n=1}^{\infty}$ such that, for all further subsequences $\left\{w_{n}\right\}_{n=1}^{\infty} \subseteq\left\{y_{n}\right\}_{n=1}^{\infty}$,

$$
n^{-1 / p} \sum_{j=1}^{n} w_{j} \in N(\varepsilon, \delta)
$$

for all sufficiently large $n \in \mathbb{N}$. The assertion of the lemma then follows from a diagonal argument, since the measure topology has a countable base of neighbourhoods at 0 .

Let $\varepsilon>0, \delta>0$ be given. Since $x_{n} \rightarrow 0$ in measure, it follows from a straightforward argument that there exists a projection $e \in \mathcal{M}$ with $\tau(\mathbf{1}-e)$ $<\delta$ and a subsequence $\left\{y_{n}\right\}_{n=1}^{\infty} \subseteq\left\{x_{n}\right\}_{n=1}^{\infty}$ such that $\left\|y_{n} e\right\|_{\infty} \leq 2^{-n-1}$ for all $n \in \mathbb{N}$. If $\left\{w_{n}\right\}_{n=1}^{\infty} \subseteq\left\{y_{n}\right\}_{n=1}^{\infty}$ is any further subsequence, then

$$
\left\|\left(n^{-1 / p} \sum_{j=1}^{n} w_{j}\right) e\right\|_{\infty} \leq n^{-1 / p}<\varepsilon
$$

for all sufficiently large $n \in \mathbb{N}$, and, together with the above remarks, this suffices to complete the proof of the lemma.

We may now state the following complement to Theorem 3.9.

TheOREM 3.11. Let $E$ be $p$-convex and $q$-concave for some $1<p<2 \leq$ $q<\infty$. If $\left\{x_{n}\right\}_{n=1}^{\infty} \subseteq E(\mathcal{M}, \tau)$ is weakly null, then there exists a subsequence $\left\{y_{n}\right\}_{n=1}^{\infty} \subseteq\left\{x_{n}\right\}_{n=1}^{\infty}$ such that $n^{-1 / p} \sum_{j=1}^{n} w_{j} \rightarrow 0$ in measure for all further subsequences $\left\{w_{n}\right\}_{n=1}^{\infty} \subseteq\left\{y_{n}\right\}_{n=1}^{\infty}$.

Proof. By the same argument as in the final paragraph of the proof of Theorem 2.14, we may assume in addition that $(\mathcal{M}, \tau)$ is non-atomic. It will suffice to show that if $\varepsilon>0$ is given and $N(\varepsilon, \varepsilon)$ is the corresponding neighbourhood of 0 in $\widetilde{\mathcal{M}}$ for the measure topology, then there exists a subsequence $\left\{y_{n}\right\}_{n=1}^{\infty} \subseteq\left\{x_{n}\right\}_{n=1}^{\infty}$ such that the sequence $\left\{n^{-1 / p} \sum_{j=1}^{n} w_{j}\right\}_{n=1}^{\infty}$ is eventually in $N(\varepsilon, \varepsilon)$ for all further subsequences $\left\{w_{n}\right\}_{n=1}^{\infty} \subseteq\left\{y_{n}\right\}_{n=1}^{\infty}$. If this is the case, then the assertion of the theorem will follow from a diagonal argument, since the measure topology has a countable base at 0 .

From Proposition 2.7, we may assume that

$$
x_{n}=y_{n}+z_{n}, \quad n \in \mathbb{N}
$$

where $\left\{y_{n}\right\}_{n=1}^{\infty}$ is equimeasurable and $z_{n} \rightarrow 0$ in measure. Since $E$ is reflexive, it follows that $E(\mathcal{M}, \tau)$ is reflexive [DDP3]. Passing to a subsequence if necessary, we may assume that $\left\{z_{n}\right\}_{n=1}^{\infty}$ is relatively weakly compact. By Lemma 2.9, we may assume that $\left\{z_{n}\right\}_{n=1}^{\infty}$, and so also $\left\{y_{n}\right\}_{n=1}^{\infty}$, is weakly null. By Lemma 3.10 applied to $\left\{z_{n}\right\}_{n=1}^{\infty}$, we may assume that $n^{-1 / p} \sum_{j=1}^{\infty} z_{j} \rightarrow 0$ in measure. We may therefore assume further that $\left\{x_{n}\right\}_{n=1}^{\infty}$ is equimeasurable so that $\mu\left(x_{n}\right)=\mu\left(x_{1}\right)$ for all $n \in \mathbb{N}$. It is also 
clear that we may assume $x_{n}=x_{n}^{*}$ for all $n \in \mathbb{N}$. By the continuity of the embedding of $E(\mathcal{M}, \tau)$ into $\widetilde{\mathcal{M}}$, there exists $\eta>0$ such that

$$
x \in E(\mathcal{M}, \tau),\|x\|_{E(\mathcal{M}, \tau)}<\eta \Rightarrow x \in N(\varepsilon / 2, \varepsilon / 2) .
$$

As observed earlier, the given assumptions on $E$ imply that $E(\mathcal{M}, \tau)$ has type $p$, with constant $M_{p}$. Since $E$ is separable, there exist $0<\delta<\Delta<\infty$ such that

$$
\left\|\mu\left(x_{1}\right)\left(\chi_{(0, \delta)}+\chi_{[\Delta, \infty)}\right)\right\|_{E(\mathcal{M}, \tau)}<\eta / 16 M_{p} .
$$

As in the proof of Theorem 3.9, there exists a sequence $\left\{e_{n}\right\}_{n=1}^{\infty} \subseteq \mathcal{M}$ of projections such that $x_{n} e_{n}=e_{n} x_{n}$ for all $n \in \mathbb{N}$ and such that

$$
\begin{aligned}
\mu\left(x_{n} e_{n}\right) & =\mu\left(\mu\left(x_{1}\right) \chi_{[\delta, \Delta)}\right), \\
\mu\left(x_{n}\left(\mathbf{1}-e_{n}\right)\right) & =\mu\left(\mu\left(x_{1}\right)\left(\chi_{(0, \delta)}+\chi_{[\Delta, \infty)}\right)\right)
\end{aligned}
$$

for all $n \in \mathbb{N}$. Since $\left\{x_{n} e_{n}\right\}_{n=1}^{\infty}$ is equimeasurable, it is simultaneously bounded, and hence relatively weakly compact, in $E(\mathcal{M}, \tau)$ and in $L_{2}(\mathcal{M}, \tau)$. Since $\left\{x_{n}\right\}_{n=1}^{\infty}$ is weakly null, and passing to a further subsequence if necessary, we may assume that there exists $a \in E(\mathcal{M}, \tau) \cap L_{2}(\mathcal{M}, \tau)$ such that $\left\{x_{n} e_{n}\right\}_{n=1}^{\infty}$ converges weakly to $a$ in $E(\mathcal{M}, \tau)$ and in $L_{2}(\mathcal{M}, \tau)$, and such that $\left\{x_{n}\left(\mathbf{1}-e_{n}\right)\right\}_{n=1}^{\infty}$ converges weakly in $E(\mathcal{M}, \tau)$ to $-a$. By (3.24) and (3.25), observe that

$$
\begin{aligned}
\|a\|_{E(\mathcal{M}, \tau)} & \leq \liminf _{n \rightarrow \infty}\left\|x_{n}\left(\mathbf{1}-e_{n}\right)\right\|_{E(\mathcal{M}, \tau)} \\
& =\lim _{n \rightarrow \infty}\left\|x_{n}\left(\mathbf{1}-e_{n}\right)\right\|_{E(\mathcal{M}, \tau)}<\eta / 16 M_{p} .
\end{aligned}
$$

By passing to a further subsequence, we may assume that the weakly null sequence $\left\{x_{n}\left(\mathbf{1}-e_{n}\right)+a\right\}_{n=1}^{\infty}$ satisfies the assertion of Lemma 3.8. Given $n \geq 1$, let $k \in \mathbb{N}$ satisfy $2^{k-1} \leq n<2^{k}$, and observe that

$$
\begin{aligned}
& \frac{1}{n^{1 / p}}\left\|\sum_{j=1}^{n}\left(x_{j}\left(\mathbf{1}-e_{j}\right)+a\right)\right\|_{E(\mathcal{M}, \tau)} \\
& \quad \leq \frac{1}{n^{1 / p}}\left\|\sum_{j=1}^{k-1}\left(x_{j}\left(\mathbf{1}-e_{j}\right)+a\right)\right\|_{E(\mathcal{M}, \tau)}+\frac{1}{n^{1 / p}}\left\|\sum_{j=k}^{n}\left(x_{j}\left(\mathbf{1}-e_{j}\right)+a\right)\right\|_{E(\mathcal{M}, \tau)} \\
& \quad \leq \frac{\ln n}{n^{1 / p} \ln 2} 2 \eta+\frac{1}{n^{1 / p}}\left\|\sum_{j=k}^{n}\left(x_{j}\left(\mathbf{1}-e_{j}\right)+a\right)\right\|_{E(\mathcal{M}, \tau)} \\
& \quad<\eta / 2+\frac{1}{n^{1 / p}}\left\|\sum_{j=k}^{n}\left(x_{j}\left(\mathbf{1}-e_{j}\right)+a\right)\right\|_{E(\mathcal{M}, \tau)}
\end{aligned}
$$

for all sufficiently large $n \in \mathbb{N}$. Using now the fact that the sequence $\left\{x_{j}\left(\mathbf{1}-e_{j}\right)+a\right\}_{j=k}^{2^{k}}$ is 4 -unconditional, from (3.24)-(3.26) and the fact that 
$E(\mathcal{M}, \tau)$ is of type $p$ with constant $M_{p}$, we obtain

$$
\begin{aligned}
& \frac{1}{n^{1 / p}}\left\|\sum_{j=k}^{n}\left(x_{j}\left(\mathbf{1}-e_{j}\right)+a\right)\right\|_{E(\mathcal{M}, \tau)} \\
& \quad \leq \frac{4 M_{p}}{n^{1 / p}}\left(\sum_{j=k}^{n}\left\|x_{j}\left(\mathbf{1}-e_{j}\right)+a\right\|_{E(\mathcal{M}, \tau)}^{p}\right)^{1 / p} \\
& \quad \leq 4 M_{p} \max _{k \leq j \leq n}\left\|x_{j}\left(\mathbf{1}-e_{j}\right)+a\right\|_{E(\mathcal{M}, \tau)} \leq 4 M_{p} 2 \eta / 16 M_{p}<\eta / 2 .
\end{aligned}
$$

It now follows that

$$
\frac{1}{n^{1 / p}}\left\|\sum_{j=1}^{n}\left(x_{j}\left(\mathbf{1}-e_{j}\right)+a\right)\right\|_{E(\mathcal{M}, \tau)}<\eta
$$

for all sufficiently large $n \in \mathbb{N}$. This in turn implies that

$$
\frac{1}{n^{1 / p}} \sum_{j=1}^{n}\left(x_{j}\left(\mathbf{1}-e_{j}\right)+a\right) \in N(\varepsilon / 2, \varepsilon / 2)
$$

for all sufficiently large $n \in \mathbb{N}$. By Proposition 3.2, the space $L_{2}(\mathcal{M}, \tau)$ has the 2-Banach-Saks property, and so there exists a constant $C(\delta, \Delta)>0$ such that

$$
n^{-1 / 2}\left\|\sum_{j=1}^{n}\left(x_{j} e_{j}-a\right)\right\|_{L_{2}(\mathcal{M}, \tau)} \leq C(\delta, \Delta)
$$

for all $n \in \mathbb{N}$. Thus,

$$
n^{-1 / p}\left\|\sum_{j=1}^{n}\left(x_{j} e_{j}-a\right)\right\|_{L_{2}(\mathcal{M}, \tau)} \leq C(\delta, \Delta) n^{1 / 2-1 / p}
$$

for all $n \in \mathbb{N}$ and this implies that $n^{-1 / p} \sum_{j=1}^{n}\left(x_{j} e_{j}-a\right) \rightarrow 0$ in $L_{2}(\mathcal{M}, \tau)$ and hence also for the measure topology. Consequently,

$$
n^{-1 / p} \sum_{j=1}^{n}\left(x_{j} e_{j}-a\right) \in N(\varepsilon / 2, \varepsilon / 2)
$$

for all sufficiently large $n \in \mathbb{N}$. Together with (3.28), this shows that

$$
n^{-1 / p} \sum_{j=1}^{n} x_{j} \in N(\varepsilon, \varepsilon)
$$

for all sufficiently large $n \in \mathbb{N}$, and this completes the proof of the theorem.

If $X \subseteq E(\mathcal{M}, \tau)$ is a closed linear subspace, then a sequence $\left\{x_{n}\right\}_{n=1}^{\infty}$ $\subseteq X$ is said to be almost disjointly supported if there exists a sequence 
$\left\{y_{n}\right\}_{n=1}^{\infty} \subseteq E(\mathcal{M}, \tau)$ which is right and left disjointly supported such that $\left\|x_{n}-y_{n}\right\|_{E(\mathcal{M}, \tau)} \rightarrow 0$ as $n \rightarrow \infty$.

Proposition 3.12. Suppose that $E$ is p-convex and $q$-concave for some $1<p<2 \leq q<\infty$, that $(\mathcal{M}, \tau)$ is non-atomic and that $X \subseteq E(\mathcal{M}, \tau)$ is a closed linear subspace. If $X$ does not have the strong p-Banach-Saks property, then $X$ contains a seminormalised almost disjointly supported sequence which converges to zero in measure.

Proof. Suppose that $\left\{x_{j}\right\}_{j=1}^{\infty} \subseteq X$ is a weakly null sequence which contains no strong $p$-Banach-Saks subsequence. Passing to subsequences if necessary, and appealing to [Ran2], we may assume that

$$
x_{j}=y_{j}+z_{j}, \quad j \geq 1,
$$

where $\left\{y_{j}\right\}_{j=1}^{\infty},\left\{z_{j}\right\}_{j=1}^{\infty}$ are bounded sequences such that $\left\{y_{j}\right\}_{j=1}^{\infty}$ is $E$-equiintegrable and $\left\{z_{j}\right\}_{j=1}^{\infty}$ is two-sided disjointly supported. Since $E(\mathcal{M}, \tau)$ is reflexive, we may assume that $\left\{z_{j}\right\}_{j=1}^{\infty}$ is weakly convergent, and hence weakly null by Lemma 2.4. It follows that we may now also assume that $\left\{y_{j}\right\}_{j=1}^{\infty}$ is weakly null. By Theorem 3.11, we may assume that $n^{-1 / p} \sum_{k=1}^{n} x_{k} \rightarrow 0$ for the measure topology. By Theorem 3.9, we may assume further that $\left\{y_{j}\right\}_{j=1}^{\infty}$ is a strong $p$-Banach-Saks sequence. It follows that $\left\{z_{n}\right\}_{n=1}^{\infty}$ contains no strong $p$-Banach-Saks subsequence. Let $\varepsilon_{k} \downarrow 0$. There exists $\delta>0$ and an increasing sequence $n(k) \uparrow \infty$ such that

$$
\begin{gathered}
n(k)^{-1 / p}\left\|\sum_{j=1}^{n(k)} z_{j}\right\|_{E(\mathcal{M}, \tau)} \geq \delta, \quad k \geq 1, \\
n(k)^{-1 / p} \max \left\{\left\|\sum_{j=1}^{n(k)} y_{j}\right\|_{E(\mathcal{M}, \tau)},\left\|\sum_{j=1}^{n(k-1)} z_{j}\right\|_{E(\mathcal{M}, \tau)}\right\} \leq \varepsilon_{k}, \quad k \geq 1 .
\end{gathered}
$$

It follows from (3.29) and (3.30) that

$$
n(k)^{-1 / p}\left\|\sum_{j=n(k-1)+1}^{n(k)} z_{j}\right\|_{E(\mathcal{M}, \tau)} \geq \delta / 2, \quad k \geq 1 .
$$

We define the sequence $\left\{w_{k}\right\}_{k=1}^{\infty} \subseteq E(\mathcal{M}, \tau)$ by setting

$$
w_{k}:=n(k)^{-1 / p} \sum_{j=n(k-1)+1}^{n(k)} z_{j}, \quad k \geq 1 .
$$

It is clear that $\left\{w_{k}\right\}_{k=1}^{\infty}$ is two-sided disjointly supported. As shown in [CDS, Lemma 2.6], there exists a pairwise disjointly supported sequence $\left\{f_{n}\right\}_{n=1}^{\infty} \subseteq E$ such that

$$
\mu\left(f_{n}\right)=\mu\left(z_{n}\right), \quad n \geq 1 .
$$


It follows readily that

$$
\mu\left(\sum_{j=1}^{n} c_{j} f_{j}\right)=\mu\left(\sum_{j=1}^{n}\left|c_{j}\right|\left|z_{j}\right|\right)=\mu\left(\sum_{j=1}^{n} c_{j} z_{j}\right)
$$

for every $n \geq 1$ and every finite sequence $c_{1}, \ldots, c_{n}$ of scalars. Since $E$ is $p$-convex, we obtain

$$
\begin{aligned}
& \left\|w_{k}\right\|_{E(\mathcal{M}, \tau)} \\
= & n(k)^{-1 / p}\left\|\sum_{j=n(k-1)+1}^{n(k)} z_{j}\right\|_{E(\mathcal{M}, \tau)}=n(k)^{-1 / p}\left\|\sum_{j=n(k-1)+1}^{n(k)} f_{j}\right\|_{E} \\
\leq & n(k)^{-1 / p}\left(\sum_{j=n(k-1)+1}^{n(k)}\left\|\mu\left(z_{j}\right)\right\|_{E}^{p}\right)^{1 / p} \leq M_{p} \sup _{j \in \mathbb{N}}\left\|z_{j}\right\|_{E(\mathcal{M}, \tau)}, \quad k \in \mathbb{N} .
\end{aligned}
$$

It follows from (3.31), (3.32) that $\left\{w_{n}\right\}_{n=1}^{\infty} \subseteq E(\mathcal{M}, \tau)$ is seminormalised. If we now set

$$
u_{n}:=n^{-1 / p} \sum_{j=1}^{n} x_{j}, \quad n \geq 1,
$$

then the sequence $\left\{u_{n}\right\}_{n=1}^{\infty} \subset X$ converges to zero for the measure topology and $\left\|u_{n}-w_{n}\right\|_{E(\mathcal{M}, \tau)} \rightarrow 0$. This suffices to complete the proof of the proposition.

We now consider the case that $E$ is a Lorentz space $\Lambda_{\psi, p}=\Lambda_{\psi, p}[0, \alpha)$, $1 \leq p<\infty$, on some interval $[0, \alpha), 0<\alpha \leq \infty$. We suppose that $\psi$ is a positive concave function on $[0, \infty)$ with $\psi(0+)=0$ and $\psi(\infty)=\infty$. The Lorentz space $\Lambda_{\psi, p}$ is the space of all measurable functions $f$ on the interval $[0, \alpha)$ such that

$$
\|f\|_{\psi, p}=\left(\int_{[0, \alpha)} \mu(f)^{p} d \psi\right)^{1 / p}<\infty .
$$

From [KPS, Lemma II.5.1], the space $\Lambda_{\psi, p}$ is separable, and it follows directly that $\Lambda_{\psi, p}$ has the Fatou property. Further, since $\Lambda_{\psi, p}$ is the $p$ convexification [LT] of the 1-convex Banach lattice $\Lambda_{\psi, 1}=\Lambda_{\psi}$, it follows that $\Lambda_{\psi, p}$ is $p$-convex. The Köthe dual $\Lambda_{\psi}^{\times}$is the Marcinkiewicz space $M_{\psi}$ consisting of all measurable functions $f$ on $[0, \alpha)$ such that

$$
\|f\|_{M_{\psi}}=\sup _{0<t<\alpha} \psi(t)^{-1} \int_{0}^{t} \mu_{s}(f) d s<\infty .
$$

It is easy to see that if $\alpha=\infty$, then $L_{\infty} \subseteq M_{\psi}=\Lambda_{\psi}^{\times}$if and only if $\lim _{t \rightarrow \infty} \psi(t) / t>0$. Equivalently, $\Lambda_{\psi}^{\times} \subseteq L_{0}$ if and only if $\lim _{t \rightarrow \infty} \psi(t) / t=0$. The concave function $\psi$ will be called regular if there exists a constant $k>1$ 
such that $\psi(2 t)>k \psi(t)$ for all $t>0$. It is shown in [Re] (see also [Dil], [No]) that $\Lambda_{\psi, p}$ is $q$-concave for some $q<\infty$ if and only if $\psi$ is regular.

While various special cases of the lemma which follows are known, we include details for the sake of completeness.

LEMmA 3.13. Let $\left\{x_{n}\right\}_{n=1}^{\infty} \subseteq \Lambda_{\psi, p}[0, \alpha)$ be seminormalised and disjointly supported. If $\alpha<\infty$, or if $\alpha=\infty$ and $x_{n} \rightarrow 0$ in measure, then $\left\{x_{n}\right\}_{n=1}^{\infty}$ contains a subsequence equivalent to the unit vector basis of $l_{p}$.

Proof. In the case that $\alpha<\infty$, the lemma is proved in [FJT, Theorem 5.1]. The argument in the case that $\alpha=\infty$ is similar. It is well known (cf. [KPS, Lemma II 2.1 and Theorem II 3.4]) that if $x \in \Lambda_{\psi, p}$, then

$$
\|x\|_{\Lambda_{\psi, p}}=\sup \left(\int_{0}^{\infty}|x \circ \sigma(t)|^{p} d \psi(t)\right)^{1 / p}
$$

where the supremum is taken over all measure-preserving mappings $\sigma$ of $[0, \infty)$ into itself. If $A \subseteq[0, \infty)$ is any measurable subset, we denote by $|A|$ the Lebesgue measure of $A$. We may assume that $\left\|x_{n}\right\|_{\Lambda_{\psi, p}} \leq 1$ for all $n \in \mathbb{N}$. Let $\varepsilon_{n} \downarrow 0$. We may assume that $\left|\left\{\left|x_{n}\right|>\varepsilon_{n}\right\}\right|<\varepsilon_{n}$. We set

$$
y_{n}=x_{n} \chi_{\left\{\left|x_{n}\right|>\varepsilon_{n}\right\}}, \quad z_{n}=x_{n}-y_{n}, \quad n \in \mathbb{N} .
$$

It is clear that $\operatorname{supp} y_{n}<\varepsilon_{n}$ and $\left|z_{n}\right| \leq \varepsilon_{n}$ a.e. for all $n \in \mathbb{N}$. We may assume that inf $\left\|y_{n}\right\|_{\Lambda_{\psi, p}}>2 \delta$ and that inf $\left\|z_{n}\right\|_{\Lambda_{\psi, p}}>2 \delta$, for some $\delta>0$. By separability of $E$, we may assume that $z_{n}$ is compactly supported and we set $s_{n}=\operatorname{supp} z_{n}$ for all $n \in \mathbb{N}$. Observing that

$$
\left\|z_{n}\right\|_{\Lambda_{\psi, p}} \leq \varepsilon_{n} \psi\left(s_{n}\right)^{1 / p}, \quad n \in \mathbb{N},
$$

we may assume that $s_{n} \uparrow_{n} \infty$. Passing to a subsequence and relabelling if necessary, we may assume there exist measure-preserving transformations

$$
\sigma_{n}:\left[0, \varepsilon_{n}\right) \rightarrow \operatorname{supp} y_{n}, \quad \omega_{n}:\left[0, s_{n}\right) \rightarrow \operatorname{supp} z_{n}
$$

such that

$$
\int_{\left[\varepsilon_{n+1}, \varepsilon_{n}\right)}\left|y_{n} \circ \sigma_{n}(t)\right|^{p} d \psi(t)>\delta^{p}, \quad \int_{\left[s_{n-1}, s_{n}\right)}\left|z_{n} \circ \omega_{n}(t)\right|^{p} d \psi(t)>\delta^{p}
$$

for all $n \in \mathbb{N}$. It is clear that we may assume that $\varepsilon_{1}<s_{1}$. Let now $\sigma$ be any measure-preserving transformation on $[0, \infty)$ such that $\sigma$ coincides with $\sigma_{n}$ on $\left[\varepsilon_{n+1}, \varepsilon_{n}\right)$ and with $\omega_{n}$ on $\left[s_{n-1}, s_{n}\right)$ for all $n \in \mathbb{N}$. Let $\left\{a_{n}\right\}_{n=1}^{N}$ be any finite scalar sequence. We obtain 


$$
\begin{aligned}
\| \sum_{n=1}^{N} a_{n} & \left.x_{n}\right|_{\Lambda_{\psi, p}} ^{p} \\
& \geq \int_{0}^{\infty}\left|\sum_{n=1}^{N} a_{n} x_{n} \circ \sigma(t)\right|^{p} d \psi(t) \\
& \geq \sum_{n=1}^{N}\left|a_{n}\right|^{p} \int_{\varepsilon_{n+1}}^{\varepsilon_{n}}\left|x_{n} \circ \sigma_{n}(t)\right|^{p} d \psi(t)+\sum_{n=1}^{N}\left|a_{n}\right|^{p} \int_{s_{n-1}}^{s_{n}}\left|x_{n} \circ \omega_{n}(t)\right|^{p} d \psi(t) \\
& =\sum_{n=1}^{N}\left|a_{n}\right|^{p} \int_{\varepsilon_{n+1}}^{\varepsilon_{n}}\left|y_{n} \circ \sigma_{n}(t)\right|^{p} d \psi(t)+\sum_{n=1}^{s_{n}}\left|a_{n}\right|^{p}\left|z_{n} \circ \omega_{n}(t)\right|^{p} d \psi(t) \\
& \geq 2 \delta^{p}\left(\sum_{n=1}^{N}\left|a_{n}\right|^{p}\right) .
\end{aligned}
$$

On the other hand, since $\Lambda_{\psi, p}$ is $p$-convex and therefore satisfies an upper $p$-estimate, we deduce that

$$
\left\|\sum_{n=1}^{N} a_{n} x_{n}\right\|_{\Lambda_{\psi, p}} \leq\left(\sum_{n=1}^{N}\left|a_{n}\right|^{p}\right)^{1 / p},
$$

and this suffices to conclude the proof.

THEOREM 3.14.

(i) If $1 \leq p<\infty$, then $\Lambda_{\psi, p}(\mathcal{M}, \tau)$ has the Banach-Saks property for all semifinite $(\mathcal{M}, \tau)$.

(ii) If $1<p<2$ and if $\psi$ is regular then, for all semifinite $(\mathcal{M}, \tau)$,

(a) $\Lambda_{\psi, p}(\mathcal{M}, \tau)$ has the $p$-Banach-Saks property;

(b) a closed linear subspace $X \subseteq \Lambda_{\psi, p}(\mathcal{M}, \tau)$ has the strong $p$-BanachSaks property if and only if $X$ contains no subspace isomorphic to $l_{p}$

(c) if $(\mathcal{M}, \tau)$ is non-atomic, then a weakly null sequence $\left\{x_{j}\right\}_{j=1}^{\infty} \subseteq$ $\Lambda_{\psi, p}(\mathcal{M}, \tau)$ which is $\Lambda_{\psi, p}$-equiintegrable has a strong $p$-BanachSaks subsequence.

Proof. (i) By the commutative specialisation of Theorem 2.13 (see also [DSS, Theorem 4.5]) and by Theorem 2.14, it will suffice to show that if $\left\{x_{n}\right\}_{n=1}^{\infty} \subseteq \Lambda_{\psi, p}$ is weakly null and disjointly supported then $\left\{x_{n}\right\}_{n=1}^{\infty}$ contains a Banach-Saks subsequence. We may suppose that $\sup _{n}\left\|x_{n}\right\|_{\Lambda_{\psi, p}} \leq 1$. If $p>1$, then $\Lambda_{\psi, p}$ is $p$-convex and therefore satisfies an upper $p$-estimate. 
In this case, there exists a constant $M>0$ such that

$$
\left\|n^{-1} \sum_{j=1}^{n} x_{j}\right\|_{\Lambda_{\psi, p}} \leq M n^{1 / p-1} \rightarrow 0 .
$$

Consequently, $\left\{x_{n}\right\}_{n=1}^{\infty}$ is a Banach-Saks sequence. We may then suppose that $p=1$. If $\alpha<\infty$, or if $\alpha=\infty$ and $\lim _{t \rightarrow \infty} \psi(t) / t>0$, then it follows from [CSS, Theorem 1] that the sequence $\left\{x_{n}\right\}_{n=1}^{\infty}$ is $\Lambda_{\psi}$-equiintegrable. Since $\left\{x_{n}\right\}_{n=1}^{\infty}$ is disjointly supported, this implies that $\left\|x_{n}\right\|_{\Lambda_{\psi}} \rightarrow 0$ so that $\left\{x_{n}\right\}_{n=1}^{\infty}$ is a Banach-Saks sequence.

We may therefore assume that $\alpha=\infty$ and that $\lim _{t \rightarrow \infty} \psi(t) / t=0$. By the commutative specialisation of Proposition 2.7 (see also [DSS, Proposition 3.2]), passing to a subsequence and relabelling if necessary we set

$$
x_{n}=y_{n}+z_{n},
$$

where the sequences $\left\{y_{n}\right\}_{n=1}^{\infty},\left\{z_{n}\right\}_{n=1}^{\infty}$ are disjointly supported, $\left\{y_{n}\right\}_{n=1}^{\infty}$ is equimeasurable, and $\left\{z_{n}\right\}_{n=1}^{\infty}$ converges to 0 for the measure topology. The condition $\lim _{t \rightarrow \infty} \psi(t) / t=0$ implies that $\Lambda_{\psi}^{\times} \subseteq L_{0}[0, \infty)$ and so by Proposition 2.7(ii), we may assume that each of the sequences $\left\{y_{n}\right\}_{n=1}^{\infty},\left\{z_{n}\right\}_{n=1}^{\infty}$ is weakly null. We may also assume that $\left\{x_{n}\right\}_{n=1}^{\infty}$ is not a Banach-Saks sequence, and for ease of notation, we assume that there exists $\delta>0$ such that

$$
n^{-1}\left\|\sum_{j=1}^{n} x_{j}\right\|_{\Lambda_{\psi}}>\delta>0
$$

for all $n \in \mathbb{N}$. We set

$$
X_{n}=\frac{1}{n} \sum_{j=1}^{n} x_{j}, \quad Y_{n}=\frac{1}{n} \sum_{j=1}^{n} y_{j}, \quad Z_{n}=\frac{1}{n} \sum_{j=1}^{n} z_{j}
$$

for all $n \in \mathbb{N}$.

We show that $\left\|Y_{n}\right\|_{\Lambda_{\psi}} \rightarrow 0$. We set $f=\mu\left(y_{n}\right), n \in \mathbb{N}$, and observe that

$$
\mu_{t}\left(Y_{n}\right)=\frac{1}{n} f\left(\frac{t}{n}\right), \quad t>0, n \in \mathbb{N} .
$$

It follows that

$$
\left\|Y_{n}\right\|_{\Lambda_{\psi}}=\int_{0}^{\infty} \frac{1}{n} f\left(\frac{t}{n}\right) \psi^{\prime}(t) d t=\int_{0}^{\infty} f(s) \psi^{\prime}(n s) d s
$$

for all $n \in \mathbb{N}$. Now, since $0 \leq \psi^{\prime}$ is non-increasing,

$$
f \psi^{\prime}(n \cdot) \leq f \psi^{\prime}
$$

for all $n \in \mathbb{N}$. Since $\lim _{t \rightarrow \infty} \psi(t) / t=0$, it follows from the concavity of $\psi$ 
that $\lim _{t \rightarrow \infty} \psi^{\prime}(t)=0$. The dominated convergence theorem now implies

$$
\left\|Y_{n}\right\|_{\Lambda_{\psi}}=\int_{0}^{\infty} f(s) \psi^{\prime}(n s) d s \rightarrow 0 .
$$

It now follows from (3.33) that $\left\|Z_{n}\right\|_{\Lambda_{\psi}}>\delta / 4$ for all sufficiently large $n \in \mathbb{N}$. Let $\varepsilon_{k} \downarrow 0$. We may assume there exists an increasing sequence $n(k) \uparrow \infty$ such that

$$
n(k+1)^{-1}\left\|\sum_{j=1}^{n(k)} z_{j}\right\|_{\Lambda_{\psi}}<\varepsilon_{k}, \quad n(k+1)^{-1}\left\|\sum_{j=n(k)+1}^{n(k+1)} z_{j}\right\|_{\Lambda_{\psi}}>\frac{\delta}{8} .
$$

If we set

$$
Z_{k}^{\prime}=n(k+1)^{-1} \sum_{j=n(k)+1}^{n(k+1)} z_{j}
$$

then the sequence $\left\{Z_{n}^{\prime}\right\}_{n=1}^{\infty}$ is disjointly supported, seminormalised and converges to 0 in measure. By Lemma 3.13, $\left\{Z_{n}^{\prime}\right\}_{n=1}^{\infty}$ contains a subsequence equivalent to the unit vector basis of $l_{1}$. From this, it follows that $\left\{Z_{n}^{\prime}\right\}_{n=1}^{\infty}$ is not weakly null, and this implies that the sequence $\left\{z_{n}\right\}_{n=1}^{\infty}$ is not weakly null, which is a contradiction.

(ii)(a) This follows from Proposition 3.2 and from the remarks preceding Lemma 3.13 .

(ii)(b) Assume first that $(\mathcal{M}, \tau)$ is non-atomic. It is clear that if $X$ contains a subspace isomorphic to $l_{p}$, then $X$ does not have the strong $p$ Banach-Saks property. Conversely, if $X$ does not have the strong $p$-BanachSaks property, then it follows from Proposition 3.12 that $X$ contains a seminormalised almost disjointly supported sequence $\left\{x_{n}\right\}_{n=1}^{\infty}$ which converges to 0 in measure. The argument in the proof of Proposition 3.12 shows that the sequence $\left\{x_{n}\right\}_{n=1}^{\infty}$ is equivalent to some disjointly supported seminormalised sequence $\left\{g_{n}\right\}_{n=1}^{\infty}$ in $\Lambda_{\psi, p}$ which converges to zero in measure. That $\left\{g_{n}\right\}_{n=1}^{\infty}$, and consequently also $\left\{x_{n}\right\}_{n=1}^{\infty}$, contains a subsequence equivalent to the unit vector basis in $l_{p}$ follows from Lemma 3.13 . The assumption that $\mathcal{M}$ is non-atomic is now removed as in the proof of Theorem 2.14.

(ii)(c) This follows from Theorem 3.9 and the remarks preceding Lemma 3.13.

The preceding theorem extends several results proved in [HRS, Theorem 4.6] for the special case that $\Lambda_{\psi, p}=L_{p}[0,1]$, and appears to be new, even in the commutative setting. The assertion of (i) for $p=1$ is given in [DSS, Theorem 5.7(i)], but the proof given there is valid only in the case of finite intervals. 


\section{References}

[Ar] J. Arazy, Basic sequences, embeddings and the uniqueness of the symmetric structure in unitary matrix spaces, J. Funct. Anal. 40 (1981), 302-340.

[Ba] S. Banach, Théorie des opérations linéaires, Monograf. Mat. 1, Warszawa, 1932.

[BS] S. Banach et S. Saks, Sur la convergence forte dans les champs $L^{p}$, Studia Math. 2 (1930), 51-57.

[BD] A. Belanger and J. Diestel, A remark on weak convergence in the dual of a $C^{*}$-algebra, Proc. Amer. Math. Soc 98 (1986), 185-186.

[BeS] C. Bennett and R. Sharpley, Interpolation of Operators, Academic Press, Boston, 1988.

[BrS1] A. Brunel and L. Sucheston, On B-convex Banach spaces, Math. Systems Theory 7 (1974), 294-299.

[BrS2] - - - On J-convexity and some ergodic super-properties of Banach spaces, Trans. Amer. Math. Soc 204 (1975), 79-90.

[CDS] V. I. Chilin, P. G. Dodds and F. A. Sukochev, The Kadec-Klee property in rearrangement invariant spaces of measurable operators, Israel J. Math. 97 (1997), 203-219.

[CKS] V. I. Chilin, A. V. Krygin and F. A. Sukochev, Uniform and local uniform convexity in spaces of measurable operators, Math. Proc. Cambridge Philos. Soc. 111 (1992), 355-368.

[CSS] V. I. Chilin, A. A. Sedaev and F. A. Sukochev, Weak compactness in Lorentz spaces, Uzbek Math. J. 1 (1993), 84-93 (in Russian).

[CS] V. I. Chilin and F. A. Sukochev, Weak convergence in non-commutative symmetric spaces, J. Operator Theory 31 (1994), 35-65.

[Di] J. Diestel, Geometry of Banach Spaces - Selected Topics, Lecture Notes in Math. 485, Springer, Berlin, 1975.

[Dil] S. J. Dilworth, Special Banach lattices and their applications, in: Handbook of the Geometry of Banach Spaces, Vol. 1, W. B. Johnson and J. Lindenstrauss (eds.), Elsevier, Amsterdam, 2001, 497-532.

[DDP1] P. G. Dodds, T. K. Dodds and B. de Pagter, Non-commutative Banach function spaces, Math. Z. 201 (1989), 583-597.

[DDP2] - - - 一, Fully symmetric operator spaces, Integral Equations Operator Theory 15 (1992), 942-972.

[DDP3] - - - - Non-commutative Köthe duality, Trans. Amer. Math. Soc. 339 (1993), $717-750$.

[DScS] P. G. Dodds, G. Schlüchtermann and F. A. Sukochev, Weak compactness in rearrangement invariant operator spaces, Math. Proc. Cambridge Philos. Soc. 131 (2001), 363-384.

[DSS] P. G. Dodds, E. M. Semenov and F. A. Sukochev, The Banach-Saks property in rearrangement invariant spaces, Studia Math. 162 (2004), 263-294.

[EM] P. Erdős and M. Magidor, A note on regular methods of summability and the Banach-Saks property, Proc. Amer. Math. Soc. 59 (1976), 232-234.

[Fa] T. Fack, Type and cotype inequalities for non-commutative $L_{p}$-spaces, J. Operator Theory 17 (1987), 255-279.

[FK] T. Fack and H. Kosaki, Generalized s-numbers of $\tau$-measurable operators, Pacific J. Math. 123 (1986), 269-300.

[FJT] T. Figiel, W. B. Johnson and L. Tzafriri, On Banach lattices and spaces having local unconditional structure, with applications to Lorentz function spaces, J. Approx. Theory 13 (1975), 395-412. 
[FS] T. Figiel and L. Sucheston, An application of Ramsey sets in analysis, Adv. Math. 20 (1976), 103-105.

[Fr] D. H. Fremlin, Topological Riesz Spaces and Measure Theory, Cambridge Univ. Press, London, 1974.

[HRS] U. Haagerup, H. P. Rosenthal and F. A. Sukochev, Banach embedding properties of non-commutative $L^{p}$-spaces, Mem. Amer. Math. Soc. 163 (2003), no. 776.

[HLR] R. Haydon, M. Levy and Y. Raynaud, Randomly Normed Spaces, Travaux en Cours 41, Hermann, Paris, 1991.

[KP] M. I. Kadec and A. Pełczyński, Bases, lacunary sequences and complemented subspaces in the spaces $L_{p}$, Studia Math. 21 (1962), 161-176.

[Ko] J. Komlós, A generalization of a problem of Steinhaus, Acta Math. Acad. Sci. Hungar. 18 (1967), 217-229.

[KPS] S. G. Krein, Yu. I. Petunin and E. M. Semenov, Interpolation of Linear Operators, Transl. Math. Monogr. 54, Amer. Math. Soc., Providence, RI, 1982; Russian original: Nauka, Moscow, 1978.

[LT] J. Lindenstrauss and L. Tzafriri, Classical Banach Spaces II, Springer, Berlin, 1979.

[Ne] E. Nelson, Notes on non-commutative integration, J. Funct. Anal. 15 (1974), 103-116.

[No] S. Ya. Novikov, Cotype and type of Lorentz function spaces, Mat. Zametki 32 (1982), 213-221 (in Russian); English transl.: Math. Notes 32 (1982), 586-590.

[PSW] B. de Pagter, F. A. Sukochev and H. Witvliet, Double operator integrals, J. Funct. Anal. 192 (2002), 52-111.

[Rak] S. A. Rakov, Banach-Saks exponent of certain Banach spaces of sequences, Mat. Zametki 32 (1982), 613-625 (in Russian); English transl.: Math. Notes 32 (1982), 791-797.

[Ran1] N. Randrianantoanina, Non-commutative subsequence principles, Math. Z. 245 (2003), 625-644.

[Ran2] -, Sequences in non-commutative $L_{p}$-spaces, J. Operator Theory 48 (2002), $255-272$.

[RX] Y. Raynaud and Q. Xu, On subspaces of non-commutative $L_{p}$-spaces, J. Funct. Anal. 203 (2003), 149-196.

[Re] S. Reisner, A factorization theorem in Banach lattices and its application to Lorentz spaces, Ann. Inst. Fourier (Grenoble) 31 (1981), 239-255.

[Ro] H. Rosenthal, Weakly independent sequences and the Banach-Saks property, in: Durham Sympos. on the Relations between Infinite-Dimensional and FiniteDimensional Convexity (Durham, 1975), Bull. London Math. Soc. 8 (1976), $22-24$.

[SZ] Ş. Strătilă and L. Zsidó, Lectures on von Neumann Algebras, Editura Academiei, Bucharest, and Abacus Press, Tunbridge Wells, 1979.

[Su] F. A. Sukochev, Non-isomorphism of $L_{p}$-spaces associated with finite and infinite von Neumann algebras, Proc. Amer. Math. Soc. 124 (1996), 1517-1527.

[Sz] W. Szlenk, Sur les suites faiblement convergentes dans l'espace L, Studia Math. 25 (1965), 337-341.

[Ta] M. Takesaki, Theory of Operator Algebras I, Springer, New York, 1979.

[Te] M. Terp, $L^{p}$-spaces associated with von Neumann algebras, notes, Copenhagen Univ., 1981.

[To] E. V. Tokarev, Subspaces of certain symmetric spaces, Teor. Funktsiu Funktsional. Anal. i Prilozhen. 24 (1975), 156-161 (in Russian). 
[Xu1] Q. Xu, Convexité uniforme des espaces symétriques d'opérateurs mesurables, C. R. Acad. Sci. Paris Sér. I Math. 309 (1989), 251-254.

[Xu2] - Analytic functions with values in lattices and symmetric spaces of measurable operators, Math. Proc. Cambridge Philos. Soc. 109 (1991), 541-563.

School of Informatics and Engineering

The Flinders University of South Australia

Bedford Park, 5042, Australia

E-mail: peter,theresa,sukochev@infoeng.flinders.edu.au

Received August 1, 2005

Revised version October 25, 2006 